\title{
Copper(II) Acetate Promoted Intramolecular Diamination of Unactivated Olefins
}

\author{
Thomas P. Zabawa, Dhanalakshmi Kasi and Sherry R. Chemler* \\ Department of Chemistry, 618 Natural Sciences Complex, University at Buffalo, State \\ University of New York, Buffalo, NY 14260 schemler@buffalo.edu
}

\section{Supplementary Material}

General Experimental Information: All reagents were used as purchased from Aldrich, Acros or Strem. Copper(II) acetate was purchased from Aldrich as a fine powder (98\%). Solvents were purified using a solvent filtration system purchased from Contour Glass Co (Irvine, California). ${ }^{1} \mathrm{H}$ NMR data were obtained in $\mathrm{CDCl}_{3}$ (using 7.26 ppm for reference of residual $\mathrm{CHCl}_{3}$ ) at 300,400 or $500 \mathrm{MHz}$ using Varian instruments. ${ }^{13} \mathrm{C}$ NMR data in $\mathrm{CDCl}_{3}$ (using $77.0 \mathrm{ppm}$ as internal reference) were obtained at 75.5 or 125.5 MHz. IR spectra were obtained from thin films on $\mathrm{KBr}$ plates using a NicoletImpact 420 FTIR. High resolution mass spectra were obtained at SUNY, Buffalo's mass spec. facility on a ThermoFinnigan MAT XL spectrometer purchased by an NSF grant to the center (NSF CHE0091977).

Data for all new compounds and selected experimentals and ${ }^{1} \mathrm{H}$ NMR spectra enclosed.

\section{Synthesis of Diamination Substrates}

The sulfamide tether was introduced by reaction of the primary amine with benzylamine chlorosulfonic acid. ${ }^{1}$

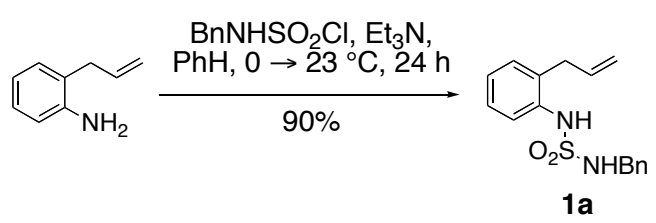

Representative Sulfamide Synthesis: $N$-(2-Allyl-phenyl)- $N^{\prime}$-benzyl-sulfamide (1a). ${ }^{2}$ A solution of $o$-Allylaniline $(3.00 \mathrm{~g}, 22.5 \mathrm{mmol})$ and triethylamine $(4.56 \mathrm{~g}, 5.98 \mathrm{~mL}$, $45.08 \mathrm{mmol})$ in dry benzene $(100 \mathrm{~mL})$ was cooled to $0{ }^{\circ} \mathrm{C}$ and treated with a $0.1 \mathrm{M}$ solution of benzylsulfonylchloride ${ }^{1}$ in benzene $(7.73 \mathrm{~g}, 37.6 \mathrm{mmol})$. After warming to room temperature, the mixture was allowed to stir for $24 \mathrm{~h}$ and the solvent was removed in vacuo. Chromatograpy of the crude oil on $\mathrm{SiO}_{2}\left(10-30 \% \mathrm{Et}_{2} \mathrm{O} /\right.$ hexanes gradient) provided $6.14 \mathrm{~g}, 90 \%$ of sulfamide $\mathbf{1 a}$ as a yellow/brown solid.

Data for 1a: $\mathrm{mp}=50-52{ }^{\circ} \mathrm{C} ;{ }^{1} \mathrm{H}$ NMR $\left(300 \mathrm{MHz}, \mathrm{CDCl}_{3}\right) \delta 7.50(\mathrm{~d}, J=8.0 \mathrm{~Hz}, 1 \mathrm{H})$, 7.29-7.11 (m, $8 \mathrm{H}), 6.43(\mathrm{~s}, 1 \mathrm{H}), 5.92(\mathrm{~m}, 1 \mathrm{H}), 5.12(\mathrm{~m}, 2 \mathrm{H}), 4.67(\mathrm{~m}, 1 \mathrm{H}), 4.22(\mathrm{~d}, J=$ $6.1 \mathrm{~Hz}, 2 \mathrm{H}), 3.37(\mathrm{~d}, J=6.0 \mathrm{~Hz}, 2 \mathrm{H}) ;{ }^{13} \mathrm{C}$ NMR $\left(75.5 \mathrm{MHz}, \mathrm{CDCl}_{3}\right) \delta 136.0,135.6$, 130.7, 130.7, 129.9, 128.8, 128.1, 127.9, 127.9, 125.2, 121.0, 117.2, 47.6, 36.5; IR (neat, thin film) 3302, 1494, 1328, 1152, 919, $751 \mathrm{~cm}^{-1}$; HRMS (EI) calcd for $[\mathrm{M}]^{+}$ $\mathrm{C}_{16} \mathrm{H}_{18} \mathrm{~N}_{2} \mathrm{O}_{2} \mathrm{~S}: 302.1089$, found 302.1084 . 
1-(2-Allyl-phenyl)-3-benzyl-urea (1b). ${ }^{1}$

The synthesis of urea $\mathbf{1 b}$ has been reported. ${ }^{2}$

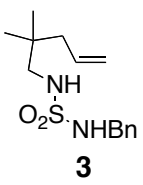

$N$-Benzyl- $N^{\prime}$-2,2-dimethylpent-4-enyl-sulfamide (3).

2,2-Dimethylpent-4-enylamine ${ }^{3,4}$ was treated with benzylsulfonylchloride in the procedure described above, providing sulfamide $\mathbf{3}$ as a white solid.

Data for 3: $\mathrm{mp}=70-74{ }^{\circ} \mathrm{C} ;{ }^{1} \mathrm{H}$ NMR $\left(500 \mathrm{MHz}, \mathrm{CDCl}_{3}\right) \delta$ 7.39-7.29 (m, $\left.5 \mathrm{H}\right), 5.76(\mathrm{~m}, 1$ H), 5.05 (m, $2 \mathrm{H}), 4.54$ (bs, $1 \mathrm{H}), 4.21$ (s, $2 \mathrm{H}), 4.18$ (bs, $1 \mathrm{H}), 2.74$ (s, $2 \mathrm{H}), 1.96$ (d, J = $7.2 \mathrm{~Hz}, 2 \mathrm{H}), 0.872(\mathrm{~s}, 6 \mathrm{H}) ;{ }^{13} \mathrm{C} \mathrm{NMR}\left(75.5 \mathrm{MHz}, \mathrm{CDCl}_{3}\right) \delta 136.7,134.3,128.8,128.0$, 127.9, 117.9, 52.8, 47.2, 44.1, 33.8, 24.8; IR (neat, thin film) 3283, 2962, 2358, 1419, 1316, 1146, 1056, $915 \mathrm{~cm}^{-1}$; HRMS (EI) calcd for $[\mathrm{M}+\mathrm{Na}]^{+} \mathrm{C}_{14} \mathrm{H}_{22} \mathrm{O}_{2} \mathrm{~N}_{2} \mathrm{SNa}$ : 305.1294, found 305.1305.

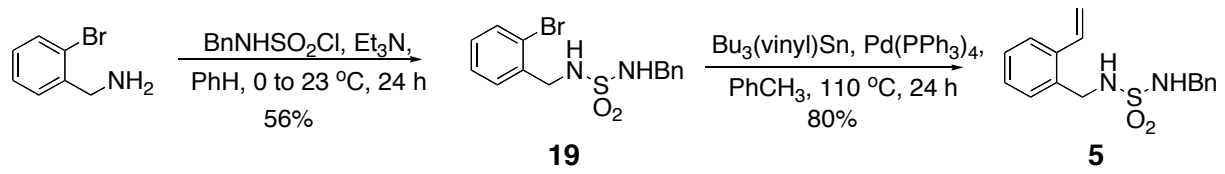

$N$-benzyl- $N^{\prime}$-(2-vinyl-benzyl)-sulfamide (5).

2-Bromobenzylamine $(0.75 \mathrm{~g}, 4.03 \mathrm{mmol})$ was treated with benzylsulfonylchloride $(1.6$ equiv) as in the procedure described above. Chromatography of the crude oil on $\mathrm{SiO}_{2}$ (20-40\% $\mathrm{Et}_{2} \mathrm{O} /$ hexanes gradient) provided $0.80 \mathrm{~g}, 56 \%$ of the brominated sulfamide 19. Data for 19: ${ }^{1} \mathrm{H}$ NMR $\left(400 \mathrm{MHz}, \mathrm{CDCl}_{3}\right) \delta 7.57(\mathrm{~d}, J=7.6,1 \mathrm{H}), 7.40-7.17(\mathrm{~m}, 8 \mathrm{H})$, 4.75 (bs. $1 \mathrm{H}), 4.42-4.29$ (m, $3 \mathrm{H}), 4.11$ (s, $2 \mathrm{H}) ;{ }^{13} \mathrm{C} \mathrm{NMR}\left(75.5 \mathrm{MHz}, \mathrm{CDCl}_{3}\right) \delta 136.9$, 136.6, 133.6, 131.1, 130.3, 129.3, 128.6, 128.4, 124.3, 48.1, 47.9; IR (neat, thin film) 3278, 2869, 1708, 1439, 1322, 1157, $1063 \mathrm{~cm}^{-1}$; HRMS (EI) calcd for $[\mathrm{M}+\mathrm{Na}]^{+}$ $\mathrm{C}_{14} \mathrm{H}_{15} \mathrm{~N}_{2} \mathrm{O}_{2} \mathrm{SNaBr}$ : 376.9930, found 376.9927.

The brominated sulfamide $19(0.24 \mathrm{~g}, 0.68 \mathrm{mmol})$ was treated with vinyltributyl tin $(0.28$ $\mathrm{g}, 0.26 \mathrm{~mL}, 0.88 \mathrm{mmol})$ and palladium tetrakistriphenylphosphine $(0.16 \mathrm{~g}, 0.14 \mathrm{mmol})$ in dry toluene $(15 \mathrm{~mL})$ and refluxed for $24 \mathrm{~h} .{ }^{5}$ The mixture was filtered through $\mathrm{SiO}_{2}$ and the solvent removed in vacuo. The crude oil was chromatographed on $\mathrm{SiO}_{2}$ (30-50\% ether/hexanes gradient) providing $0.16 \mathrm{~g}, 80 \%$ yield, of sulfamide $\mathbf{5}$ as a yellow solid. Data for 5: $\mathrm{mp}=81-84{ }^{\circ} \mathrm{C} ;{ }^{1} \mathrm{H}$ NMR $\left(300 \mathrm{MHz}, \mathrm{CDCl}_{3}\right) \delta 7.56(\mathrm{~d}, J=7.5,1 \mathrm{H}), 7.38$ $7.24(\mathrm{~m}, 8 \mathrm{H}), 7.00(\mathrm{~m}, 1 \mathrm{H}), 5.73(\mathrm{~d}, J=17.41 \mathrm{H}), 5.40(\mathrm{~d}, J=11.1,1 \mathrm{H}), 4.38$ (bs, 1 $\mathrm{H}), 4.26-4.15(\mathrm{~m}, 5 \mathrm{H}) ;{ }^{13} \mathrm{C} \mathrm{NMR}\left(75.5 \mathrm{MHz}, \mathrm{CDCl}_{3}\right) \delta 137.6,137.1,134.0,133.8,130.1$, 130.0, 129.3, 129.2, 128.7, 128.6, 126.8, 117.9, 47.8, 45.7; IR (neat, thin film) 3254, 2349, 1429, 1316, 1160, $1051 \mathrm{~cm}^{-1}$; HRMS (EI) calcd for $[\mathrm{M}+\mathrm{Na}]^{+} \mathrm{C}_{16} \mathrm{H}_{18} \mathrm{~N}_{2} \mathrm{O}_{2} \mathrm{SNa}$ : 325.0981, found 325.0980. 

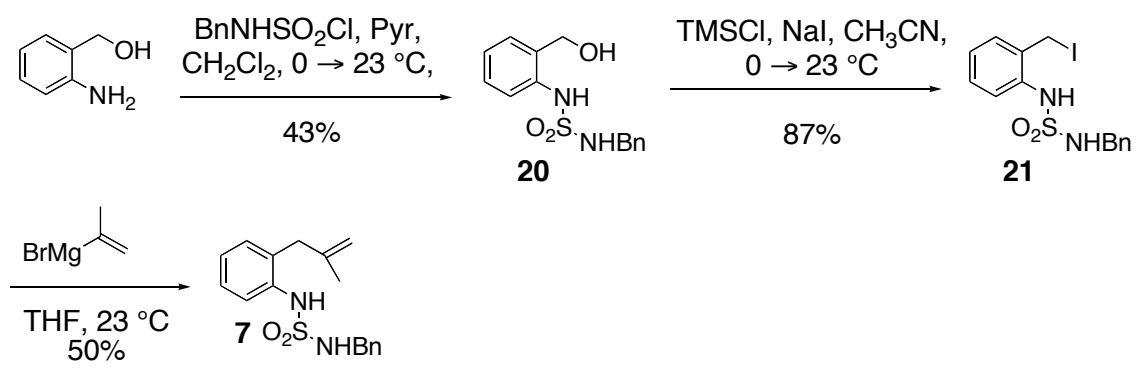

\section{$N$-Benzyl- $N^{\prime}$-(2-methyl-allyl-phenyl)-sulfamide (7).}

A solution of 2-Aminobenzylalcohol (3.86 g, $0.031 \mathrm{~mol}, 1$ equiv) and pyridine ( $3 \mathrm{~mL}$, 0.038 mol, 1.2 equiv) in $100 \mathrm{~mL}$ of $\mathrm{CH}_{2} \mathrm{Cl}_{2}$ was cooled to $0{ }^{\circ} \mathrm{C}$ and treated with benzylaminesulfonyl chloride $\left(6.4 \mathrm{~g}, 0.031 \mathrm{~mol}, 0.99\right.$ equiv) in $20 \mathrm{~mL}$ of $\mathrm{CH}_{2} \mathrm{Cl}_{2}$. After being stirred $12 \mathrm{~h}$ at $23{ }^{\circ} \mathrm{C}$, the volatiles were removed in vacuo. The crude product was dissolved in EtOAc $(100 \mathrm{~mL})$ and washed with saturated aqueous $\mathrm{NH}_{4} \mathrm{Cl}(20 \mathrm{~mL})$. The aqueous layer was extracted with EtOAc $(2 \times 100 \mathrm{~mL})$ and the combined organic layers dried over $\mathrm{Na}_{2} \mathrm{SO}_{4}$, filtered and concentrated in vacuo. The crude oil was purified by flash chromatography on $\mathrm{SiO}_{2}(30-40 \% \mathrm{EtOAc} /$ hexane eluent), providing $3.9 \mathrm{~g}$ of sulfamide $\mathbf{2 0}$ as a white solid (43\% yield).

Data for (2-sulfamido benzylalcohol 20): $\mathrm{mp} 79-81{ }^{\circ} \mathrm{C} ;{ }^{1} \mathrm{H}$ NMR $\left(400 \mathrm{MHz}, \mathrm{CDCl}_{3}\right) \delta$ $7.86(\mathrm{~s}, 1 \mathrm{H}), 7.55(\mathrm{~d}, J=8.0 \mathrm{~Hz}, 1 \mathrm{H}), 7.33(\mathrm{dd}, J=7.6,8.0 \mathrm{~Hz}, 1 \mathrm{H}), 7.25(\mathrm{~m}, 3 \mathrm{H})$, $7.16(\mathrm{~m}, 2 \mathrm{H}), 7.08$ (t, $J=7.3, \mathrm{~Hz}, 1 \mathrm{H}), 4.83$ (s, $1 \mathrm{H}), 4.66(\mathrm{~s}, 2 \mathrm{H}), 4.25$ (d, $J=5.8 \mathrm{~Hz}, 2$ $\mathrm{H}), 1.95$ (bs, $1 \mathrm{H}) ;{ }^{13} \mathrm{C}$ NMR $(75.45 \mathrm{MHz}) \delta 136.9,136.2,129.3,129.2,128.9,128.6$, 127.89, 127.86, 124.2, 120.3, 64.0, 47.4; IR (neat, thin film) 3307, 3056, 2926, 1603, $1589,1497,1455,1418,1330,1265,1153,1060,1003,934 \mathrm{~cm}^{-1}$; HRMS (EI) calcd for $[\mathrm{M}]^{+} \mathrm{C}_{14} \mathrm{H}_{16} \mathrm{~N}_{2} \mathrm{O}_{3} \mathrm{~S}_{1}: 292.0876$, found 292.08646.

Benzylalcohol 20 ( $3.0 \mathrm{~g}, 0.01 \mathrm{~mol}, 1$ equiv) in $\mathrm{CH}_{3} \mathrm{CN}(150 \mathrm{~mL})$ was cooled to $0{ }^{\circ} \mathrm{C}$ and treated with trimethylsilyl chloride $\left(3.5 \mathrm{~g}, 0.032 \mathrm{~mol}, 3.2\right.$ equiv). ${ }^{6}$ Sodium iodide ( $4.9 \mathrm{~g}$, $0.032 \mathrm{~mol}, 3.2$ equiv) was added and the mixture was stirred for $2 \mathrm{~h}$ at $0{ }^{\circ} \mathrm{C}$, then $2 \mathrm{~h}$ at 23 ${ }^{\circ} \mathrm{C}$. The reaction was quenched with ice water, extracted with $\mathrm{CH}_{2} \mathrm{Cl}_{2}(3 \times 50 \mathrm{~mL})$ and the combined organic layers were washed with $20 \mathrm{~mL}$ of saturated sodium thiosulfate solution and $50 \mathrm{~mL}$ of water. The organic layer was dried over $\mathrm{Na}_{2} \mathrm{SO}_{4}$, filtered and concentrated in vacuo to yield $3.6 \mathrm{~g}$ of iodide $\mathbf{2 1}$ as a white solid (87\% yield).

Data for iodide 21: $\mathrm{mp} 102-104{ }^{\circ} \mathrm{C} ;{ }^{1} \mathrm{H}$ NMR $\left(300 \mathrm{MHz}, \mathrm{CDCl}_{3}\right) \delta 7.46(\mathrm{~d}, J=7.9 \mathrm{~Hz}, 1$ H), 7.31-7.18 (m,7 H), $7.13(\mathrm{~m}, 1 \mathrm{H}), 6.42(\mathrm{~s}, 1 \mathrm{H}), 4.82(\mathrm{~m}, 1 \mathrm{H}), 4.32(\mathrm{~s}, 2 \mathrm{H}), 4.28(\mathrm{~d}, J$ $=6.0 \mathrm{~Hz}, 2 \mathrm{H}) ;{ }^{13} \mathrm{C}$ NMR $(75.45 \mathrm{MHz}) \delta 135.9,135.1,130.2,130.1,129.7,128.8,128.1$, 127.9, 125.6, 122.4, 47.7, 2.2; IR (neat, thin film) 3306, 3047, 3032, 1496,1410, 1329, $1153,1062,924 \mathrm{~cm}^{-1}$; HRMS (EI) calcd for $[\mathrm{M}]^{+} \mathrm{C}_{14} \mathrm{H}_{15} \mathrm{I}_{1} \mathrm{~N}_{2} \mathrm{O}_{2} \mathrm{~S}_{1}$ : 401.9893, found 401.99030

In a two neck round bottom flask, $\mathrm{Mg}(0.036 \mathrm{~g}, 0.00149 \mathrm{~mol}, 3$ equiv) in THF ( $2 \mathrm{~mL})$ was treated with 2-bromopropene $(0.18 \mathrm{~g}, 0.00149 \mathrm{~mol}, 3$ equiv), added dropwise via syringe. ${ }^{7}$ The mixture was stirred at $23{ }^{\circ} \mathrm{C}$ for $1 \mathrm{~h}$, then iodide $21(0.2 \mathrm{~g}, 0.00049,1$ equiv) in THF ( $2 \mathrm{~mL})$ was added and the mixture was stirred an additional hour. The 
reaction was quenched with saturated aqueous $\mathrm{NH}_{4} \mathrm{Cl}$ and extracted with $\mathrm{CH}_{2} \mathrm{Cl}_{2}$. The organic layer was washed with water, dried over $\mathrm{Na}_{2} \mathrm{SO}_{4}$, filtered and concentrated in vacuo. Purification of the crude residue by flash chromatography on $\mathrm{SiO}_{2}(10 \% \mathrm{EtOAc} /$ hexane) afforded $0.078 \mathrm{~g}$ of 7 (50\% yield) as a viscous, colorless oil.

Data for 7: ${ }^{1} \mathrm{H}$ NMR (400 MHz, $\left.\mathrm{CDCl}_{3}\right) \delta 7.50(\mathrm{~d}, J=7.9 \mathrm{~Hz}, 1 \mathrm{H}), 7.29-7.26(\mathrm{~m}, 4$ H), 7.18-7.12 (m, 4 H), 6.16 (s, 1 H), 4.93 (s, 1 H), 4.74 (s, $1 \mathrm{H}), 4.64$ (m, $1 \mathrm{H}), 4.20$ (d, $J=5.7 \mathrm{~Hz}, 2 \mathrm{H}), 3.33(\mathrm{~s}, 2 \mathrm{H}), 1.71(\mathrm{~s}, 3 \mathrm{H}) ;{ }^{13} \mathrm{C}$ NMR $(75.45 \mathrm{MHz}) \delta 143.9,136.3$, 136.2, 131.6, 129.5, 129.0, 128.3, 128.2, 128.2, 125.1, 120.8, 113.2, 47.7, 41.6, 22,4; IR (neat, thin film) 3305, 3067, 3030, 2976, 2920, 1648, 1586, 1495, 1454, 1331, 1153, 1097, $1064 \mathrm{~cm}^{-1}$; HRMS (ESI) calcd for $[\mathrm{M}+\mathrm{H}]^{+} \mathrm{C}_{17} \mathrm{H}_{21} \mathrm{O}_{2} \mathrm{~N}_{2} \mathrm{~S}$ : 317.1318, found 317.1309 .

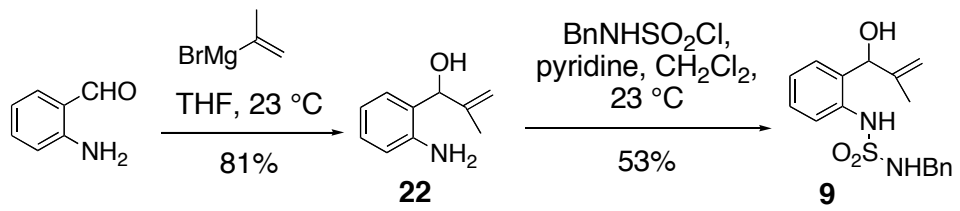

( \pm )- $N$-Benzyl- $N^{\prime}$-[2-(1-hydroxy-2-methyl-allyl)-phenyl]-sulfamide (9).

In a two neck round bottom flask, $\mathrm{Mg}(0.241 \mathrm{~g}, 0.01 \mathrm{~mol}, 3$ equiv) in THF ( $5 \mathrm{~mL})$ under Ar was treated with 2-bromopropene (1.1 g, $0.01 \mathrm{~mol}, 3$ equiv), added dropwise via syringe. The mixture was stirred at $23{ }^{\circ} \mathrm{C}$ for $1 \mathrm{~h}$ then treated with 2-aminobenzaldehyde $\left(0.4 \mathrm{~g}, 0.003,1\right.$ equiv) in THF $(5 \mathrm{~mL})$. After being stirred overnight at $23{ }^{\circ} \mathrm{C}$, the reaction was quenched with saturated aqueous $\mathrm{NH}_{4} \mathrm{Cl}$ and extracted with $\mathrm{CH}_{2} \mathrm{Cl}_{2}$. The organic layer was washed with water, dried over $\mathrm{Na}_{2} \mathrm{SO}_{4}$, filtered, and the solvent was removed in vacuo. The crude residue was purified by flash chromatography on $\mathrm{SiO}_{2}(10$ $\%$ EtOAc/ hexane), providing $0.4 \mathrm{~g}$ of allylic alcohol $\mathbf{2 2}^{\mathbf{8}}$ (81\% yield).

Aniline 22 (0.39 g, 0.0024, 1 equiv) was dissolved in $\mathrm{CH}_{2} \mathrm{Cl}_{2}(10 \mathrm{~mL})$. Pyridine $(0.19 \mathrm{ml}$, $0.0024,1$ equiv) and benzylaminesulfonyl chloride $(0.49 \mathrm{~g}, 0.0023,0.99$ equiv) were added and the solution was stirred overnight at $23{ }^{\circ} \mathrm{C}$. The reaction mixture was washed with water, dried over $\mathrm{Na}_{2} \mathrm{SO}_{4}$, and concentrated in vacuo. Purification of the crude residue by flash chromatography on $\mathrm{SiO}_{2}$ (10-20\% EtOAc / hexane as eluent) afforded $0.42 \mathrm{~g}$ of sulfamide 9 (53\% yield) as a viscous, colorless oil.

Data for 9: ${ }^{1} \mathrm{H}$ NMR $\left(400 \mathrm{MHz}, \mathrm{CDCl}_{3}\right) \delta 7.96(\mathrm{~s}, 1 \mathrm{H}), 7.57(\mathrm{~d}, J=8.0 \mathrm{~Hz}, 1 \mathrm{H}), 7.32$ $(\mathrm{dd}, J=8.7,8.0 \mathrm{~Hz}, 1 \mathrm{H}), 7.25(\mathrm{~m}, 2 \mathrm{H}), 7.15-7.08(\mathrm{~m}, 4 \mathrm{H}), 5.27(\mathrm{~s}, 1 \mathrm{H}), 5.18(\mathrm{~s}, 1 \mathrm{H})$, 5.09 (s, $1 \mathrm{H}), 4.7$ (m, $1 \mathrm{H}), 4.20$ (ddd, $J=5.49,5.49,5.86 \mathrm{~Hz}, 2 \mathrm{H}), 2.29$ (s, $1 \mathrm{H}), 1.63$ (s, $3 \mathrm{H}){ }^{13} \mathrm{C}$ NMR $(75.45 \mathrm{MHz}) \delta 144.7,136.5,136.3,129.3,129.1,128.8,128.7,127.9$, 123.7, 119.9, 111.7, 77.4, 47.4, 19.4; IR (neat, thin film) 3500, 3305, 3075, 3028, 2922, 1587, 1496, 1448, 1418, 1329, 1150, 1061, $930 \mathrm{~cm}^{-1}$; HRMS (ESI) calcd for $[\mathrm{M}+\mathrm{Na}]^{+}$ $\mathrm{C}_{17} \mathrm{H}_{20} \mathrm{~N}_{2} \mathrm{NaO}_{3} \mathrm{~S}: 355.1087$, found 355.1086 . 


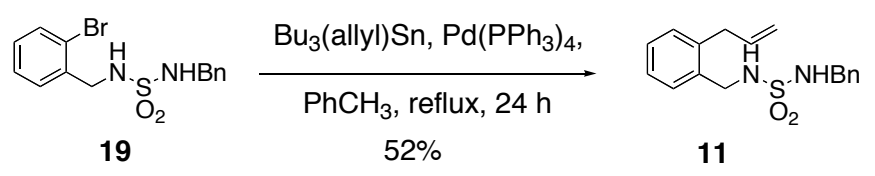

\section{$N$-(2-Allyl-benzyl)- $N^{\prime}$-benzyl-sulfamide (11).}

Brominated sulfamide $19(0.50 \mathrm{~g}, 1.40 \mathrm{mmol})$ was treated with allyltributyl tin $(0.61 \mathrm{~g}$, $0.56 \mathrm{~mL}, 1.83 \mathrm{mmol})$ and palladium tetrakistriphenylphosphine $(0.32 \mathrm{~g}(0.28 \mathrm{mmol})$ in degassed, dry toluene $(30 \mathrm{~mL})$ and the mixture was refluxed for $24 \mathrm{~h}^{.}{ }^{9}$ The crude mixture was directly filtered through $\mathrm{SiO}_{2}$ and the solvent removed in vacuo. Flash chromatography of the crude residue on $\mathrm{SiO}_{2}\left(20-50 \% \mathrm{Et}_{2} \mathrm{O} /\right.$ hexanes gradient) provided $0.23 \mathrm{~g}, 52 \%$ of sulfamide $\mathbf{1 1}$ as a white solid.

Data for 11: $\mathrm{mp}=60-64{ }^{\circ} \mathrm{C} ;{ }^{1} \mathrm{H}$ NMR $\left(500 \mathrm{MHz}, \mathrm{CDCl}_{3}\right) \delta 7.36-7.20(\mathrm{~m}, 9 \mathrm{H}), 5.96(\mathrm{~m}$, $1 \mathrm{H}), 5.09$ (dd, $J=10.0,1.5 \mathrm{~Hz}, 1 \mathrm{H}), 4.97(\mathrm{dd}, J=17.0,1.5 \mathrm{~Hz}, 1 \mathrm{H}), 4.45(\mathrm{bs}, 1 \mathrm{H})$, 4.30 (bs, $1 \mathrm{H}), 4.16$ (s, $4 \mathrm{H}), 3.44(\mathrm{~d}, J=6.0,2 \mathrm{H}) ;{ }^{13} \mathrm{C} \mathrm{NMR}\left(75.5 \mathrm{MHz}, \mathrm{CDCl}_{3}\right) \delta 138.3$, 137.0, 136.5, 134.3, 130.3, 129.5, 128.8, 128.5, 128.1, 126.9, 116.3, 47.4, 44.9, 36.8; IR (neat, thin film) 3293, 1637, 1492, 1451, 1428, 1325, 1153, 1062, 917, 749, $700 \mathrm{~cm}^{-1}$; HRMS (EI) calcd for $[\mathrm{M}+\mathrm{Na}]^{+} \mathrm{C}_{17} \mathrm{H}_{20} \mathrm{~N}_{2} \mathrm{O}_{2} \mathrm{SNa}$ : 339.1143, found 339.1130.

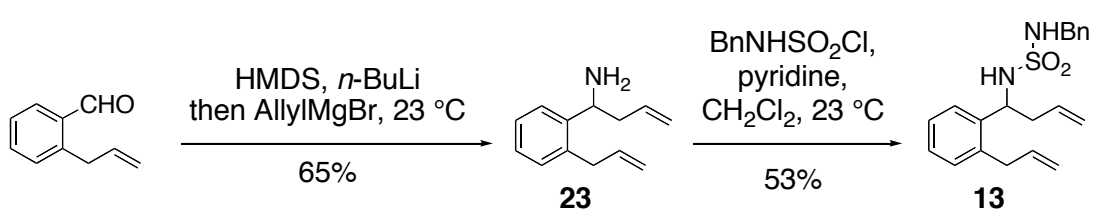

( \pm )- $N$-1-[1-(2-Allyl-phenyl)-but-3-enyl]- $N^{\prime}$-benzyl-sulfamide (13) ${ }^{10}$

2-Allylbenzaldehyde ${ }^{11}(0.536 \mathrm{~g}, 3.66 \mathrm{mmol}, 1$ equiv) in $2 \mathrm{~mL}$ of THF was treated with lithium bis(trimethylsilyl)amide prepared from 1,1,1,3,3,3-hexamethyldsilazane $(1.12 \mathrm{~g}$, $6.97 \mathrm{mmol}, 1.9$ equiv) and $1.6 \mathrm{M}$ solution of $n$-BuLi in hexanes $(3.66 \mathrm{~mL}, 5.87 \mathrm{mmol}, 1.6$ equiv) at $0{ }^{\circ} \mathrm{C}$. To the resulting solution, a $1 \mathrm{M}$ solution of allylmagnesium bromide in ether (5.8 $\mathrm{mL}, 5.87 \mathrm{mmol}, 1.6$ equiv) was added, the mixture was warmed to room temperature and stirred for $12 \mathrm{~h}$. The reaction was quenched with saturated $\mathrm{NH}_{4} \mathrm{Cl}$, extracted with $\mathrm{CH}_{2} \mathrm{Cl}_{2}$, and the combined organic layers were dried over $\mathrm{Na}_{2} \mathrm{SO}_{4}$, filtered and the solvent was removed in vacuo. Flash chromatography of the crude oil on $\mathrm{SiO}_{2}$ (30-40 \% EtOAc/Hexane) provided $0.44 \mathrm{~g}, 65 \%$ of the amine $\mathbf{2 3}$.

Data for 23: ${ }^{1} \mathrm{H}$ NMR $\left(300 \mathrm{MHz}, \mathrm{CDCl}_{3}\right) \delta 7.49(\mathrm{~d}, J=7.4 \mathrm{~Hz}, 1 \mathrm{H}), 7.27-7.13(\mathrm{~m}, 3$ H), $5.98(\mathrm{~m}, 1 \mathrm{H}), 5.75(\mathrm{~m}, 1 \mathrm{H}), 5.16-4.94(\mathrm{~m}, 4 \mathrm{H}), 4.27(\mathrm{dd}, J=7.9,5.2 \mathrm{~Hz}, 2 \mathrm{H})$, 3.53-3.38 (m, $2 \mathrm{H}), 2.50-2.30(\mathrm{~m}, 2 \mathrm{H}), 1.76$ (bs, $2 \mathrm{H}) ;{ }^{13} \mathrm{C}$ NMR $(75.45 \mathrm{MHz}) \delta 143.8$, 137.5, 136.4, 135.6, 129.8, 126.8, 125.6, 117.4, 115.6, 50.2, 43.3, 36.9; IR (neat, thin film) 3339, 3071, 2923, 1639,1549, 1444, 995, $915 \mathrm{~cm}^{-1}$; HRMS (ESI) calcd for $[\mathrm{M}+\mathrm{H}]^{+}$ $\mathrm{C}_{13} \mathrm{H}_{18} \mathrm{~N}_{1}: 188.1434$, found 188.14340 .

A solution of allylamine $23(0.1 \mathrm{~g}, 0.58 \mathrm{mmol}, 1$ equiv) and pyridine $(0.047 \mathrm{~mL}, 0.58$ mmol, 1 equiv) in $3 \mathrm{~mL}$ of $\mathrm{CH}_{2} \mathrm{Cl}_{2}$ was cooled to $0{ }^{\circ} \mathrm{C}$ and treated with a solution of benzylaminesulfonyl chloride $\left(0.12 \mathrm{~g}, 0.57 \mathrm{~mol}, 0.99\right.$ equiv) in $5 \mathrm{~mL}$ of $\mathrm{CH}_{2} \mathrm{Cl}_{2}$. After being stirred $12 \mathrm{~h}$ at $23{ }^{\circ} \mathrm{C}$, the crude product in $\mathrm{CH}_{2} \mathrm{Cl}_{2}$ was washed with water. The 
aqueous layer was extracted with $\mathrm{CH}_{2} \mathrm{Cl}_{2}$ and the combined organic layers dried over $\mathrm{Na}_{2} \mathrm{SO}_{4}$, filtered and concentrated in vacuo. The crude oil was purified by flash chromatography on $\mathrm{SiO}_{2}$ (30-40\% EtOAc/hexane eluent), providing $0.1 \mathrm{~g}$ of sulfamide (53\% yield) as a white solid.

Data for 13: $\mathrm{mp}$ 68-69 ${ }^{\circ} \mathrm{C}$; ${ }^{1} \mathrm{H}$ NMR $\left(400 \mathrm{MHz}, \mathrm{CDCl}_{3}\right) \delta$ 7.41-7.39 (m, $\left.1 \mathrm{H}\right), 7.29$-7.23 (m, 6 H), 6.97 (m, 2 H), $5.9(\mathrm{~m}, 1 \mathrm{H}), 5.73(\mathrm{~m}, 1 \mathrm{H}), 5.23-5.18(\mathrm{~m}, 2 \mathrm{H}) 5.06-4.99(\mathrm{~m}, 2$ H) $4.80(\mathrm{~m}, 1 \mathrm{H}), 4.67(\mathrm{~m}, 1 \mathrm{H}), 4.01(\mathrm{~d}, J=12.8 \mathrm{~Hz}, 1 \mathrm{H}), 3.91(\mathrm{~m}, 1 \mathrm{H}), 3.72(\mathrm{~d}, J=$ $11.7 \mathrm{~Hz}, 1 \mathrm{H}), 3.58(\mathrm{dd}, J=15.7,6.5 \mathrm{~Hz}, 1 \mathrm{H}), 3.45(\mathrm{dd}, J=16.1,5.8 \mathrm{~Hz}, 1 \mathrm{H}), 2.55-2.40$ $(\mathrm{m}, 2 \mathrm{H}) ;{ }^{13} \mathrm{C}$ NMR $(75.5 \mathrm{MHz}) \delta 139.6,137.2,136.9,136.1,133.4,130.3,128.5,128.0$, $127.8,127.7,126.9,126.3,119.5,116.2,52.3,47.1,41.7,36.9$; IR (neat, thin film) 3283, $1426,1313,1151,1061 \mathrm{~cm}^{-1}$; HRMS (ESI) calcd for $[\mathrm{M}+\mathrm{Na}]^{+} \mathrm{C}_{20} \mathrm{H}_{24} \mathrm{~N}_{2} \mathrm{NaO}_{2} \mathrm{~S}$ : 379.1451 , found 379.1447 . 


\section{Copper(II) Promoted Diamination, Table 1 Experimental Procedures}

\begin{tabular}{|c|c|c|c|c|c|}
\hline \multicolumn{3}{|c|}{$\begin{array}{l}1 \mathrm{a}, \mathrm{X}=\mathrm{SO}_{2} \\
1 \mathrm{~b}, \mathrm{X}=\mathrm{C}=\mathrm{O}\end{array}$} & \multicolumn{3}{|c|}{$\begin{array}{l}\mathbf{2 a}, \mathrm{X}=\mathrm{SO}_{2} \\
\mathbf{2 b}, \mathrm{X}=\mathrm{C}=\mathrm{O}\end{array}$} \\
\hline entry & tether & $\mathrm{Cu}^{\mathrm{n}}$ (equiv) & base (2 equiv) & solvent $^{b}$ & yield $\%^{c}$ \\
\hline 1 & $\mathrm{X}=\mathrm{SO}_{2}$ & $\mathrm{Cu}(\mathrm{OAc})_{2}(1.2$ & $\mathrm{K}_{2} \mathrm{CO}_{3}$ & THF & 43 \\
\hline 2 & $\mathrm{X}=\mathrm{SO}_{2}$ & $\mathrm{Cu}(\mathrm{OAc})_{2}(1.2$ & $\mathrm{K}_{2} \mathrm{CO}_{3}$ & $\mathrm{CH}_{3} \mathrm{CN}$ & 38 \\
\hline 3 & $\mathrm{X}=\mathrm{SO}_{2}$ & $\mathrm{Cu}(\mathrm{OAc})_{2}(1.2$ & $\mathrm{K}_{2} \mathrm{CO}_{3}$ & DMF & 78 \\
\hline 4 & $\mathrm{X}=\mathrm{SO}_{2}$ & $\mathrm{Cu}(\mathrm{OAc})_{2}(1$ & $\mathrm{K}_{2} \mathrm{CO}_{3}$ & DMF/DMSO & 92 \\
\hline 5 & $\mathrm{X}=\mathrm{C}=\mathrm{O}$ & $\mathrm{Cu}(\mathrm{OAc})_{2}(1.2$ & 2) $\mathrm{K}_{2} \mathrm{CO}_{3}$ & DMF/DMSO & no rxn \\
\hline 6 & $\mathrm{X}=\mathrm{SO}_{2}$ & $\mathrm{Cu}(\mathrm{OAc})_{2}(1.2$ & 2) $\mathrm{Cs}_{2} \mathrm{CO}_{3}$ & DMF/DMSO & 84 \\
\hline 7 & $\mathrm{X}=\mathrm{SO}_{2}$ & $\mathrm{Cu}(\mathrm{OAc})_{2}(1.2$ & none & DMF/DMSO & 34 \\
\hline 8 & $\mathrm{X}=\mathrm{SO}_{2}$ & $\mathrm{Cu}(\mathrm{OTf})_{2}(1.2$ & $\mathrm{K}_{2} \mathrm{CO}_{3}$ & DMF/DMSO & no rxn ${ }^{d}$ \\
\hline 9 & $\mathrm{X}=\mathrm{SO}_{2}$ & $\mathrm{CuF}_{2}(1.2)$ & $\mathrm{K}_{2} \mathrm{CO}_{3}$ & DMF/DMSO & no $\mathrm{rxn}^{d}$ \\
\hline 10 & $\mathrm{X}=\mathrm{SO}_{2}$ & CuOAc (1.0) & $\mathrm{K}_{2} \mathrm{CO}_{3}$ & DMF/DMSO & 22 \\
\hline 11 & $\mathrm{X}=\mathrm{SO}_{2}$ & CuOAc (3.0) & $\mathrm{K}_{2} \mathrm{CO}_{3}$ & DMF/DMSO & 54 \\
\hline
\end{tabular}

aPressure tube, $\mathrm{Ar}_{(\mathrm{g})}$ atmosphere, dry solvent. ${ }^{b} 10$ equiv DMSO.

${ }^{c}$ Remainder of material is recovered s.m. ${ }^{d}$ Some decomposition of s.m. observed.

\section{Diamination of Sulfamide 1a and Urea 1b (General Procedure for Table 1, entries 1-} 11)

Sulfamide 1a or urea $\mathbf{1 b}^{1}$ was treated with 1.0-3.0 equivalents of the appropriate copper salt, 2 equivalents of base (if used) in dry solvent, and was heated to $90{ }^{\circ} \mathrm{C}$ under an $\operatorname{Ar}(\mathrm{g}$ ) atmosphere in a pressure tube for $48 \mathrm{~h}$. The mixture was then diluted with $\mathrm{Et}_{2} \mathrm{O}$, plug filtered through $\mathrm{SiO}_{2}$, and concentrated in vacuo. The crude mixture was chromatographed on $\mathrm{SiO}_{2}\left(20-40 \% \mathrm{Et}_{2} \mathrm{O} /\right.$ hexanes gradient) providing the product 2a in the given yields.

Optimized diamination of 1a: 2-Benzyl-1,2,8,8a-tetrahydro-3-thia-2,3a-diazacyclopenta $[a]$ indene 3,3-dioxide (2a) (Table 1, entry 4).

Sulfamide 1a $(0.0357 \mathrm{~g}, 0.118 \mathrm{mmol})$ was treated with $\mathrm{Cu}(\mathrm{OAc})_{2}(0.0257 \mathrm{~g}, 0.142$ $\mathrm{mmol}), \mathrm{K}_{2} \mathrm{CO}_{3}(0.0326 \mathrm{~g}, 0.236 \mathrm{mmol})$ and DMSO (0.921 g, $\left.0.08 \mathrm{~mL}, 1.18 \mathrm{mmol}\right)$ in $\operatorname{DMF}(1.2 \mathrm{~mL})$, and the mixture heated to $90{ }^{\circ} \mathrm{C}$ under an $\operatorname{Ar}(\mathrm{g})$ atmosphere in a pressure tube, for $48 \mathrm{~h}$. The mixture was then diluted with $\mathrm{Et}_{2} \mathrm{O}$, plug filtered through $\mathrm{SiO}_{2}$, concentrated in vacuo and chromatographed on $\mathrm{SiO}_{2}\left(20-40 \% \mathrm{Et}_{2} \mathrm{O} /\right.$ hexanes gradient) to give $0.0018 \mathrm{~g}, 6 \%$ of starting material 1a (eluted first), and $0.0326 \mathrm{~g}, 92 \%$ of diamination product $\mathbf{2 a}$ as a yellow/brown solid. Note: Larger scale diamination reactions require removal of DMF by dissolving the crude material in $\mathrm{Et}_{2} \mathrm{O}$ and washing the organic solution with with water. The $\mathrm{Et}_{2} \mathrm{O}$ layer is then dried $\left(\mathrm{MgSO}_{4}\right)$, filtered and concentrated in vacuo and the product further purified as described above.

Data for sulfamide 2a: ${ }^{1} \mathrm{H}$ NMR $(500 \mathrm{MHz}) \delta$ 7.36-7.10 (m, $\left.9 \mathrm{H}\right), 4.71(\mathrm{~m}, 1 \mathrm{H}), 4.32(\mathrm{~A}$ of $\left.\mathrm{ABq}, J_{\mathrm{AB}}=13.7 \mathrm{~Hz}, 1 \mathrm{H}\right), 4.10\left(\mathrm{~B}\right.$ of ABq, $\left.J_{\mathrm{AB}}=13.7 \mathrm{~Hz}, 1 \mathrm{H}\right), 3.39(\mathrm{dd}, J=9.7,7.0$ $\mathrm{Hz}, 1 \mathrm{H}), 3.33$ (dd, $J=16.2,9.2 \mathrm{~Hz}, 1 \mathrm{H}), 3.14(\mathrm{dd}, J=16.2,5.5 \mathrm{~Hz}, 1 \mathrm{H}), 3.03(\mathrm{dd}, J=$ $15.8,5.2 \mathrm{~Hz}, 1 \mathrm{H}) ;{ }^{13} \mathrm{C}$ NMR $(125.7 \mathrm{MHz}) \delta 142.8,134.9,128.8,128.2,128.1,125.1$, 124.9, 117.8, 59.4, 52.4, 50.7, 35.7; IR (neat, thin film) 3063, 3031, 2924, 2880, 1476, 
1337, 1162, 938, $757 \mathrm{~cm}^{-1}$; HRMS (FAB) calcd for $\left[\mathrm{M}^{+}\right] \mathrm{C}_{16} \mathrm{H}_{16} \mathrm{~N}_{2} \mathrm{O}_{2} \mathrm{~S}: 300.0932$, found: 300.0930

Table 2. Intramolecular Diamination of Olefins ${ }^{a}$

entry substrate

aReaction conditions: 3 equiv $\mathrm{Cu}(\mathrm{OAc})_{2}, 2$ equiv $\mathrm{K}_{2} \mathrm{CO}_{3}$, DMF $(0.08-0.1 \mathrm{M})$, DMSO (10 equiv), $90^{\circ} \mathrm{C}, 48 \mathrm{~h}$. ${ }^{b}$ Selectivity determined by analysis of the crude ${ }^{1} \mathrm{H}$ NMR spectrum, and by amount of the isolated adducts. ${ }^{C}$ Yield refers to amount of product isolated by chromatography on silica gel. ${ }^{d}$ Reaction was conducted at $120^{\circ} \mathrm{C}$.

(士)-2-Benzyl-5,5-dimethyl-hexahydro-1-thia-2,6a-diaza-pentalene 1,1-dioxide (4), (Table 2, entry 1).

Sulfamide 3 (0.0390 g, $0.138 \mathrm{mmol})$ was treated with $\mathrm{Cu}(\mathrm{OAc})_{2}(0.0753 \mathrm{~g}, 0.414 \mathrm{mmol})$, $\mathrm{K}_{2} \mathrm{CO}_{3}(0.0382 \mathrm{~g}, 0.276 \mathrm{mmol})$ and DMSO $(0.1078 \mathrm{~g}, 0.1 \mathrm{~mL} 1.38 \mathrm{mmol})$ in DMF (1.4 $\mathrm{mL})$, and the mixture heated to $120{ }^{\circ} \mathrm{C}$ under an $\operatorname{Ar}(\mathrm{g})$ atmosphere in a pressure tube, for $48 \mathrm{~h}$. The mixture was then diluted with $\mathrm{Et}_{2} \mathrm{O}$, plug filtered through $\mathrm{SiO}_{2}$, concentrated in vacuo, and chromatographed on $\mathrm{SiO}_{2}$ (10-30\% EtOAc/hexanes gradient) to give $0.004 \mathrm{~g}$, $10 \%$ of starting material 3 (eluted first), and $0.028 \mathrm{~g}, 73 \%$ of diamination product 4 as a yellow, oily solid.

Data for sulfamide 4: ${ }^{1} \mathrm{H}$ NMR (400 $\mathrm{MHz}, \mathrm{CDCl}_{3}$ ) $\delta$ 7.36-7.26 (m $5 \mathrm{H}$ ), 4.43 (A of ABq, $\left.J_{\mathrm{AB}}=13.2 \mathrm{~Hz}, 1 \mathrm{H}\right), 4.06(\mathrm{~m} 1 \mathrm{H}), 3.81\left(\mathrm{~B}\right.$ of ABq, $\left.J_{\mathrm{AB}}=13.2 \mathrm{~Hz}, 1 \mathrm{H}\right), 3.27(\mathrm{~d} . J=9.2$ 
$\mathrm{Hz}, 1 \mathrm{H}), 3.14(\mathrm{~m}, 2 \mathrm{H}), 2.96(\mathrm{dd}, J=9.2,2.4 \mathrm{~Hz}, 1 \mathrm{H}), 7.83(\mathrm{q}, \mathrm{K}=12.0,6.4 \mathrm{~Hz}, 1 \mathrm{H})$, $1.46(\mathrm{t}, \mathrm{K}=10.4,1 \mathrm{H}), 1.17$ (s, $3 \mathrm{H}), 1.12(\mathrm{~s}, 3 \mathrm{H}) ;{ }^{13} \mathrm{C} \mathrm{NMR}\left(75.5 \mathrm{MHz}, \mathrm{CDCl}_{3}\right) \delta 235.8$, 129.3, 129.1, 128.6, 63.3, 58.8, 51.4, 50.7, 46.8, 40.9, 26.6, 26.5; IR (neat, thin film) $2950,2859,1451,1325,1167 \mathrm{~cm}^{-1}$; HRMS (EI) calcd for $[\mathrm{M}]^{+} \mathrm{C}_{14} \mathrm{H}_{20} \mathrm{~N}_{2} \mathrm{O}_{2} \mathrm{~S}$ : 280.1245, found 280.1249

( \pm )-2-Benzyl-2,3,3a,8-tetrahydro-1-thia-2,8a-diaza-cyclopenta[a]indene 1,1-dioxide (6), (Table 2, entry 2).

Sulfamide $5(0.0495 \mathrm{~g}, 0.164 \mathrm{mmol})$ was treated with $\mathrm{Cu}(\mathrm{OAc})_{2}(0.0891 \mathrm{~g}, 0.491 \mathrm{mmol})$, $\mathrm{K}_{2} \mathrm{CO}_{3}(0.0452 \mathrm{~g}, 0.327 \mathrm{mmol})$ and DMSO $(0.1281 \mathrm{~g}, 0.11 \mathrm{~mL} 1.64 \mathrm{mmol})$ in DMF (1.7 $\mathrm{mL}$ ), and the mixture heated to $120^{\circ} \mathrm{C}$ under an $\mathrm{Ar}$ atmosphere in a pressure tube, for 48 h. The mixture was then diluted with $\mathrm{Et}_{2} \mathrm{O}$, plug filtered through $\mathrm{SiO}_{2}$, concentrated in vacuo, and chromatographed on $\mathrm{SiO}_{2}\left(10-30 \% \mathrm{Et}_{2} \mathrm{O} /\right.$ hexanes gradient) to give a mixture of products. Prepratory TLC provided $0.0275 \mathrm{~g}, 56 \%$ of diamination product 6 as a yellow, oily solid.

Data for sulfamide 6: ${ }^{1} \mathrm{H}$ NMR $\left(400 \mathrm{MHz}, \mathrm{CDCl}_{3}\right) \delta$ 7.36-7.20 (m, $\left.8 \mathrm{H}\right), 7.10(\mathrm{~d}, J=7.6,1 \mathrm{H})$, $5.16(\mathrm{~m} 1 \mathrm{H}), 5.13 \mathrm{~A}$ of $\mathrm{ABq}, J=15.61 \mathrm{H}), 4.54\left(\mathrm{~B}\right.$ of ABq, $\left.J_{\mathrm{AB}}=15.2,1 \mathrm{H}\right), 4.37(\mathrm{~A}$ of ABq, $\left.J_{\mathrm{AB}}=13.6,1 \mathrm{H}\right), 3.45(\mathrm{~B}$ of $\mathrm{ABq}, J=13.6,1 \mathrm{H}), 3.50(\mathrm{dd}, J=9.6,7.6,1 \mathrm{H}), 3.31(\mathrm{dd}, J=9.2$, 3.2, $1 \mathrm{H}) ;{ }^{13} \mathrm{C}$ NMR $\left(75.5 \mathrm{MHz}, \mathrm{CD}_{2} \mathrm{Cl}_{2}\right)$ \& 141.5, 139.8, 137.4, 131.1, 131.0, 130.9, 130.5, 130.4, 124.9, 124.1, 66.3, 58.7, 55.2, 53.0; IR (neat, thin film) 2913, 1851, 2360, 2324, 1731, 1647, 1453, 1328, 1161, $1069 \mathrm{~cm}^{-1}$; HRMS (EI) calcd for $[\mathrm{M}+\mathrm{H}]^{+} \mathrm{C}_{16} \mathrm{H}_{17} \mathrm{~N}_{2} \mathrm{O}_{2} \mathrm{~S}: 301.1005$, found 301.1005 .

( \pm )-2-Benzyl-8a-methyl-1,2,8,8a-tetrahydro-3-thia-2,3a-diaza-cyclopenta[ $a]$ indene 3,3-dioxide (8), (Table 2, entry 3).

Data for 8: viscous, colorless oil, ${ }^{1} \mathrm{H}$ NMR (400 MHz, $\left.\mathrm{CDCl}_{3}\right) \delta$ 7.34-7.26 (m, $\left.7 \mathrm{H}\right), 7.18$ $-7.12(\mathrm{~m}, 2 \mathrm{H}), 4.40\left(\mathrm{~A}\right.$ of $\left.\mathrm{ABq}, J_{\mathrm{AB}}=13.1 \mathrm{~Hz}, 1 \mathrm{H}\right), 3.96\left(\mathrm{~B}\right.$ of $\mathrm{ABq}, J_{\mathrm{AB}}=13.5 \mathrm{~Hz}, 1$ H), $3.30(\mathrm{~d}, J=17.2 \mathrm{~Hz}, 1 \mathrm{H}), 3.26(\mathrm{~d}, J=9.9 \mathrm{~Hz}, 1 \mathrm{H}), 3.07(\mathrm{~d}, J=9.5 \mathrm{~Hz}, 1 \mathrm{H}), 2.94$ $(\mathrm{d}, J=16.1 \mathrm{~Hz}, 1 \mathrm{H}), 1.56(\mathrm{~s}, 3 \mathrm{H}) ;{ }^{13} \mathrm{C}$ NMR $(75.5 \mathrm{MHz}) \delta 142.4,134.8,131.0,128.8$, $128.6,128.2,128.0,125.4,124.7,118.9,68.5,57.8,50.4,43.9,27.4$; IR (neat, thin film) 2924, 2858, 1460, 1396, 1340, 1163, 1028, $901 \mathrm{~cm}^{-1}$; HRMS (ESI) calcd for $[\mathrm{M}+\mathrm{H}]^{+}$ $\mathrm{C}_{17} \mathrm{H}_{19} \mathrm{~N}_{2} \mathrm{O}_{2} \mathrm{~S}: 315.1162$, found 315.1163 .

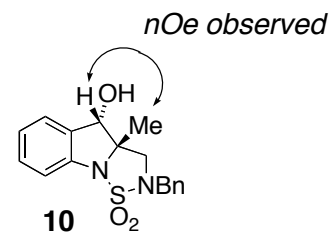

( \pm )-(8S,8aS)-2-Benzyl-8a-methyl-3,3-dioxo-2,3,8,8a-tetrahydro-1H-3 $\gamma^{6}$-thia-2,3adiaza-cyclopenta[a]inden-8-ol (10), (Table 2, entry 4).

Data for 10: viscous, colorless oil, ${ }^{1} \mathrm{H}$ NMR $\left(400 \mathrm{MHz}, \mathrm{CDCl}_{3}\right) \delta ; 7.48(\mathrm{~d}, J=7.6 \mathrm{~Hz}, 1$ $\mathrm{H}), 7.42(\mathrm{~d}, J=7.6 \mathrm{~Hz}, 1 \mathrm{H}), 7.36-7.31(\mathrm{~m}, 3 \mathrm{H}), 7.30-7.23(\mathrm{~m}, 4 \mathrm{H}), 4.50(\mathrm{~s}, 1 \mathrm{H}), 4.41$ (A of $\left.\mathrm{ABq}, J_{\mathrm{AB}}=13.1 \mathrm{~Hz}, 1 \mathrm{H}\right), 3.87\left(\mathrm{~B}\right.$ of $\left.\mathrm{ABq}, J_{\mathrm{AB}}=13.1 \mathrm{~Hz}, 1 \mathrm{H}\right), 3.82(\mathrm{~A}$ of $\mathrm{ABq}$, $\left.J_{\mathrm{AB}}=9.8 \mathrm{~Hz}, 1 \mathrm{H}\right), 2.98\left(\mathrm{~B}\right.$ of $\left.\mathrm{ABq}, J_{\mathrm{AB}}=9.8 \mathrm{~Hz}, 1 \mathrm{H}\right), 1.43(\mathrm{~s}, 3 \mathrm{H}) ;{ }^{13} \mathrm{C}$ NMR $(75.45$ MHz) $\delta 142.3,134.2,132.9,130.7,128.8,128.7,128.4,126.2,126.1,119.4,79.5,70.9$, 
51.0, 50.9, 25.3; IR (neat, thin film) 3497, 3033, 2972, 2922, 2863, 1604, 1479, 1462 , 1337, 1168, 1121, 1050, 940, $915 \mathrm{~cm}^{-1}$; HRMS (ESI) calcd for $[\mathrm{M}+\mathrm{Na}]^{+} \mathrm{C}_{17} \mathrm{H}_{18} \mathrm{~N}_{2} \mathrm{NaO}_{3} \mathrm{~S}$ : 353.0930, found 353.0938. The relative stereochemistry of $\mathbf{1 0}$ was assigned by the presence of a strong nOe signal between the benzylic hydrogen and the angular methyl.

( \pm )-2-Benzyl-3,3a,4,9-tetrahydro-2H-1-thia-2,9a-diaza-cyclopenta[b]naphthalene 1,1-dioxide (12), (Table 2, entry 5).

Sulfamide 11 (0.0179 g, $0.0565 \mathrm{mmol})$ was treated with $\mathrm{Cu}(\mathrm{OAc})_{2}(0.0308 \mathrm{~g}, 0.170$ $\mathrm{mmol}), \mathrm{K}_{2} \mathrm{CO}_{3}(0.0156 \mathrm{~g}, 0.113 \mathrm{mmol})$ and DMSO (0.0442 $\mathrm{g}, 0.04 \mathrm{~mL} 0.5650 \mathrm{mmol}$ ) in DMF $(0.6 \mathrm{~mL})$, and the mixture heated to $120{ }^{\circ} \mathrm{C}$ under an $\mathrm{Ar}$ atmosphere in a pressure tube, for $48 \mathrm{~h}$. The mixture was then diluted with $\mathrm{Et}_{2} \mathrm{O}$, plug filtered through $\mathrm{SiO}_{2}$, concentrated in vacuo, and chromatographed on $\mathrm{SiO}_{2}\left(20-40 \% \mathrm{Et}_{2} \mathrm{O} /\right.$ hexanes gradient) proviing $0.0147 \mathrm{~g}, 83 \%$ of diamination product $\mathbf{1 2}$ as a pale yellow oily solid.

Data for sulfamide 12: ${ }^{1} \mathrm{H}$ NMR $\left(400 \mathrm{MHz}, \mathrm{CDCl}_{3}\right) \delta 7.38-7.10(\mathrm{~m}, 9 \mathrm{H}), 4.52$ (A of $\mathrm{ABq}, J=14.8,1 \mathrm{H}), 4.45\left(\mathrm{~A}\right.$ of $\left.\mathrm{ABq}, J_{\mathrm{AB}}=14.0,1 \mathrm{H}\right), 4.29\left(\mathrm{~B}\right.$ of $\left.\mathrm{ABq}, J_{\mathrm{AB}}=15.2,1 \mathrm{H}\right)$, $4.09\left(\mathrm{~B}\right.$ of $\left.\mathrm{ABq}, J_{\mathrm{AB}}=14.0,1 \mathrm{H}\right), 3.63(\mathrm{~m}, 1 \mathrm{H}), 3.44(\mathrm{~m}, 1 \mathrm{H}), 3.00-2.90(\mathrm{~m}, 3 \mathrm{H}) ;{ }^{13} \mathrm{C}$ $\operatorname{NMR}\left(75.5 \mathrm{MHz}, \mathrm{CDCl}_{3}\right) \delta 137.0,130.4,129.5,128.9,128.6,128.5,128.1,127.1,126.9$, 116.3, 51.9, 44.6, 33.6, 29.7; IR (neat, thin film) 3312, 2924, 2360, 1636, 1454. 1313, 1150; HRMS (EI) calcd for [M+1] ${ }^{+} \mathrm{C}_{17} \mathrm{H}_{19} \mathrm{~N}_{2} \mathrm{O}_{2} \mathrm{~S}$ : 315.1167, found 315.1162.

(士)-9-Allyl-2-benzyl-3,3a,4,9-tetrahydro-2H-1-thia-2,9a-diazacyclopenta[b]naphthalene 1,1-dioxide, (Table 2 , entry 6).

Data for 14: viscous, colorless oil, ${ }^{1} \mathrm{H}$ NMR (400 MHz, $\left.\mathrm{CDCl}_{3}\right) \delta 7.41-7.32(\mathrm{~m}, 5 \mathrm{H})$, $7.25(\mathrm{~m}, 2 \mathrm{H}), 7.14(\mathrm{~m}, 1 \mathrm{H}), 7.07(\mathrm{~d}, J=7.7 \mathrm{~Hz}, 1 \mathrm{H}), 5.60(\mathrm{~m}, 1 \mathrm{H}), 5.04-4.87(\mathrm{~m}, 2 \mathrm{H})$, $4.60\left(\mathrm{~A}\right.$ of $\left.\mathrm{ABq}, J_{\mathrm{AB}}=14.0 \mathrm{~Hz}, 1 \mathrm{H}\right), 3.94\left(\mathrm{~B}\right.$ of $\left.\mathrm{ABq}, J_{\mathrm{AB}}=14.0 \mathrm{~Hz}, 1 \mathrm{H}\right), 3.53(\mathrm{~m}, 1$ H), $3.34(\mathrm{dd}, J=8.3,5.2 \mathrm{~Hz}, 1 \mathrm{H}), 3.16(\mathrm{~m}, 1 \mathrm{H}), 2.93(\mathrm{dd}, J=9.8,8.5 \mathrm{~Hz}, 1 \mathrm{H}), 2.83-$ $2.78(\mathrm{~m}, 3 \mathrm{H}) ;{ }^{13} \mathrm{C}$ NMR $(75.5 \mathrm{MHz}) \delta 135.0,134.8,132.6,132.1,128.76,128.7,128.1$, 127.0, 126.6, 126.5, 118.7, 56.6, 53.1, 51.5, 51.2, 38.8, 34.2; IR (neat, thin film) 3067, $3030,2918,2852,1494,1454,1305,1168,910 \mathrm{~cm}^{-1}$; HRMS (ESI) calcd for $[\mathrm{M}+\mathrm{H}]^{+}$ $\mathrm{C}_{20} \mathrm{H}_{23} \mathrm{~N}_{2} \mathrm{O}_{2} \mathrm{~S}: 355.1475$, found 355.14734.

Data for 15: viscous, colorless oil, ${ }^{1} \mathrm{H}$ NMR (400 MHz, $\left.\mathrm{CDCl}_{3}\right) \delta 7.32(\mathrm{~m}, 4 \mathrm{H}), 7.22(\mathrm{~m}$, $4 \mathrm{H}), 7.07(\mathrm{~d}, J=7.3 \mathrm{~Hz}, 1 \mathrm{H}) 5.88(\mathrm{~m}, 1 \mathrm{H}), 5.17(\mathrm{dd}, J=15.7,1.4 \mathrm{~Hz}, 2 \mathrm{H}), 4.84(\mathrm{dd}, J=$ $6.2,6.5 \mathrm{~Hz}), 4.42\left(\mathrm{~A}\right.$ of $\left.\mathrm{ABq}, J_{\mathrm{AB}}=13.9 \mathrm{~Hz}, 1 \mathrm{H}\right), 4.02(\mathrm{~m}, 1 \mathrm{H}), 3.96\left(\mathrm{~B}\right.$ of $\mathrm{ABq}, J_{\mathrm{AB}}=$ $14.2 \mathrm{~Hz}, 1 \mathrm{H}), 3.32(\mathrm{dd}, J=6.5,6.2 \mathrm{~Hz}, 1 \mathrm{H}), 3.04(\mathrm{dd}, J=9.5,9.5 \mathrm{~Hz}, 1 \mathrm{H}), 3.00$ (dd, $J=$ 2.9, 3.2 Hz, 1H), $2.80(\mathrm{dd}, J=16.1,4.7,1 \mathrm{H}), 2.66(\mathrm{t}, J=6.5 \mathrm{~Hz}, 2 \mathrm{H}) ;{ }^{13} \mathrm{C}$ NMR $(75.45$ $\mathrm{MHz}) \delta 135.18,135.12,134.2,131.7,129.2,128.7,128.5,128.0,127.1,126.9,126.8$, 118.0, 53.9, 50.8, 50.6, 48.3, 41.7, 31.4; IR (neat, thin film) 3067, 3030, 2926, 2858, $1492,1450,1297,1167,1116,1057,919 \mathrm{~cm}^{-1}$; HRMS (ESI) calcd for $[\mathrm{M}+\mathrm{H}]^{+}$ $\mathrm{C}_{20} \mathrm{H}_{23} \mathrm{~N}_{2} \mathrm{O}_{2} \mathrm{~S}: 355.1475$, found 355.14850.

The relative stereochemistry of $\mathbf{1 4}$ and $\mathbf{1 5}$ was assigned by ROESY experiments. 


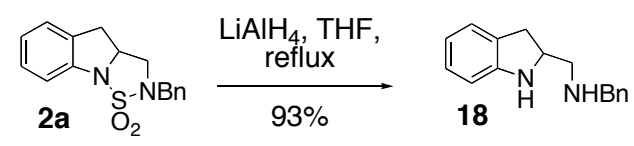

(土)-Benzyl-(2,3-dihydro-1H-indol-2-ylmethyl)-amine (18).

Using a modified procedure ${ }^{12}$, sulfonamide $2 \mathrm{a}(0.059$ g. $0.196 \mathrm{mmol})$ was treated with lithium aluminum hydride $(0.022 \mathrm{~g}, 0.588 \mathrm{mmol})$ in $\mathrm{Et}_{2} \mathrm{O}(4 \mathrm{~mL})$. The mixture was refluxed for $24 \mathrm{~h}$, then quenched with water, and finally with $15 \% \mathrm{NaOH}$. This mixture was then filtered, and the organic layer removed. The aqueous layer was extracted with $\mathrm{Et}_{2} \mathrm{O}$ three times, the organic layers combined, dried and concentrated in vacuo to provide the crude product. Chromatography on $\mathrm{SiO}_{2}\left(30-60 \% \mathrm{Et}_{2} \mathrm{O} /\right.$ hexanes gradient) provided $0.044 \mathrm{~g}, 93 \%$, of the diamine $\mathbf{1 8}$ as a yellow oil.

Data for diamine 18: ${ }^{1} \mathrm{H}$ NMR (400 MHz) $\delta$ 7.21-7.18 (m, $\left.5 \mathrm{H}\right), 7.00(\mathrm{~d}, J=7.2 \mathrm{~Hz}, 1 \mathrm{H})$, $6.95(\mathrm{t}, J=7.2 \mathrm{~Hz}, 1 \mathrm{H}), 6.63$ (t, $J=7.6 \mathrm{~Hz}, 1 \mathrm{H}), 6.56(\mathrm{~d}, J=7.6 \mathrm{~Hz}, 1 \mathrm{H}), 4.18$ (bs, 1 H) $3.88(\mathrm{~m}, 1 \mathrm{H}), 3.76(\mathrm{~s}, 2 \mathrm{H}), 3.08(\mathrm{dd}, J=15.6,9.2,1 \mathrm{H}), 2.72-2.61(\mathrm{~m}, 3 \mathrm{H}), 1.61(\mathrm{bs}$, $1 \mathrm{H}) ;{ }^{13} \mathrm{C}$ NMR $(125.7 \mathrm{MHz}) \delta 150.6,140.0,128.6,128.4,128.1,127.3,127.0,124.8$, 118.7, 109.8, 58.5, 54.3, 53.8, 33.9; IR (neat, thin film) 3360, 3027, 2921, 2839, 1606, $1483,1461,1249,746 \mathrm{~cm}^{-1}$; HRMS (FAB) calcd for $[\mathrm{M}+1]^{+} \mathrm{C}_{16} \mathrm{H}_{19} \mathrm{~N}_{2}: 239.1470$, found 239.1550 .

Mechanism Probe. The mechanism of the $\mathrm{Cu}(\mathrm{OAc})_{2}$ promoted diamination and the related aminocarbonation reaction ${ }^{13}$ are still under investigation. We do not, however, believe that a primary carbocation, e.g. 27, is involved, since the $N$-mesyl-orthoallylaniline substrate $\mathbf{2 4}$ cyclizes to provide the hydroamination adduct $\mathbf{2 6}$ in $61 \%$ yield. This compound must be obtained from a reaction intermediate through capture of either $\mathrm{H}+\mathrm{or} \mathrm{H} \bullet$. Were a primary carbocation involved, a nucleophile, such as -OAc, would have captured the carbon. Also, no products due to carbocation rearrangement were observed. Further investigation of the reaction mechanism is in progress.
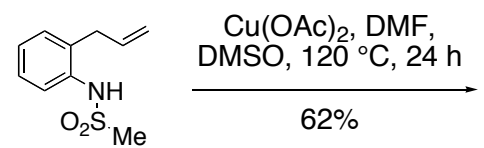

24

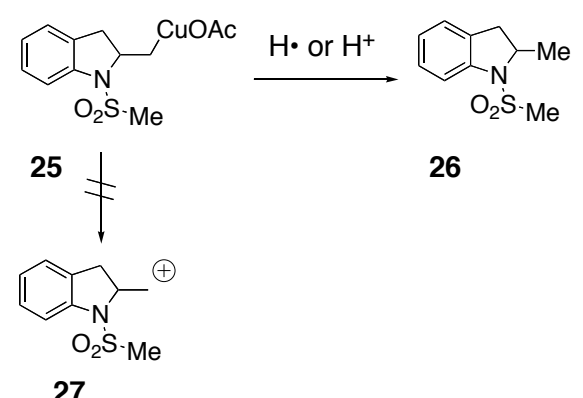

$N$-mesyl- $O$-allylaniline $24(0.1 \mathrm{~g}, 0.5 \mathrm{mmol})$ was treated with $\mathrm{Cu}(\mathrm{OAc})_{2}(0.18 \mathrm{~g}, 1.5$ $\mathrm{mmol}$ ) in $6.3 \mathrm{ml}$ of DMF and the mixture was heated to $120^{\circ} \mathrm{C}$ under Ar atmosphere in a pressure tube for $24 \mathrm{~h}$. The mixture was diluted with $\mathrm{Et}_{2} \mathrm{O}$, plug filtered through celite, concentrated in vacuo, and chromatographed on $\mathrm{SiO}_{2}$ (10 \% EtOAc/hexane), providing $0.062 \mathrm{~g}(62 \%)$ of $\mathbf{2 6}$ as a white solid.

Data for 26: mp 57-58 ${ }^{\circ} \mathrm{C} ;{ }^{1} \mathrm{H}$ NMR $\left(300 \mathrm{MHz}, \mathrm{CDCl}_{3}\right) \delta 7.41(\mathrm{~d}, J=8.2 \mathrm{~Hz}, 1 \mathrm{H}), 7.20$ (t, $J=7.1 \mathrm{~Hz}, 2 \mathrm{H}), 7.05$ (t, $J=7.4 \mathrm{~Hz}, 1 \mathrm{H}), 4.45(\mathrm{~m}, 1 \mathrm{H}), 3.45(\mathrm{dd}, J=16.2,9.6 \mathrm{~Hz}, 1$ $\mathrm{H}), 2.84(\mathrm{~s}, 3 \mathrm{H}), 2.70(\mathrm{dd}, J=16.2,3.5,1 \mathrm{H}), 1.45(\mathrm{~d}, J=6.45 \mathrm{~Hz}, 3 \mathrm{H}) \quad ;{ }^{13} \mathrm{C}$ NMR 
(75.45 MHz) $\delta$ 141.0. 130.5, 127.9, 125.5, 124.1, 115.2, 58.8, 36.5, 36.3, 23.3; IR (neat, thin film) 2967, 2929, 1601, 1479, 1460, 1344, 1249, 1226, 1158, 1103, 1003, 961, 767 $\mathrm{cm}^{-1}$; HRMS (EI) calcd for $[\mathrm{M}]^{+} \mathrm{C}_{10} \mathrm{H}_{13} \mathrm{~N}_{1} \mathrm{O}_{2} \mathrm{~S}_{1}: 211.0662$, found 211.06688 .

\section{References}

(1) (a) Audrieth, L. F.; Sveda, M. J. Org. Chem. 1944, 9, 89. (b) Kloek, J. A.; Leschinsky, K. L. J. Org. Chem. 1976, 41, 4028. Other methods for the synthesis of sulfamides: (c) Alcaraz, L.; Bennion, C.; Morris, J.; Meghani, P.; Thom, S. M. Org. Lett. 2004, 6, 2705. (d) Masui, T.; Kabaki, M.; Watanabe, H.; Kobayashi, T.; Masui, Y. Org. Proc. Res. \& Develop. 2004, 8, 408.

(2) Manzoni, M. R.; Zabawa, T. P.; Kasi, D.; Chemler, S. R. Organometallics, 2004, 23, 5618.

(3) Kimpe, N. D.; Smaele, D. D.; Hofkens, A.; DeJaegher, Y.; Kesteleyn, B. Tetrahedron, 1997, 53, 10803.

(4) Toshimitsu, A.; Terao, K.; Uemura, S. J. Org. Chem. 1986, 51, 1724.

(5) Wipf, P.; Weiner, W. S. J. Org. Chem. 1999, 64, 5321.

(6) Olah, G. A.; Husain, A.; Sing, B. P.; Mehrotra, A. K. J. Org. Chem. 1983, 48, 3667.

(7) Larock, R. C.; Pace, P.; Yang, H.; Russell, C. E. Tetrahedron, 1998, 54, 9961.

(8) Mulzer, J.; Langer, O.; Hiersemann, M.; Bats, J. W.; Buschmann, J.; Luger, P.; J. Org. Chem. 2000, 65, 6540.

(9) Ha-Duong, N.; DiJols, S.; Marques-Soares, C.; Minoletti, C.; Dansette, P. M.; Mansuy D. J. Med. Chem. 2001, 44, 3622.

(10) Hart, D. J.; Kanai, K.; Thomas, D. G.; Yang, T. J. Org. Chem. 1983, 48, 289.

(11) Kampmeier, J. A.; Harris, S. H.; Mergelsberg, I. J. Org. Chem. 1984, 49, 621625.

(12) Hendrikson, J. B.; Bergeron, R.; Sternbach, D. D. Tetrahedron. 1975, 31, 2517.

(13) Sherman, E. S.; Chemler, S. R.; Tan, T. B.; Gerlits, O. Org. Lett. 2004, 6, 1573 .

Selected ${ }^{1} \mathrm{H}$ NMR and nOe spectra follow: 
S 13

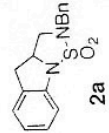

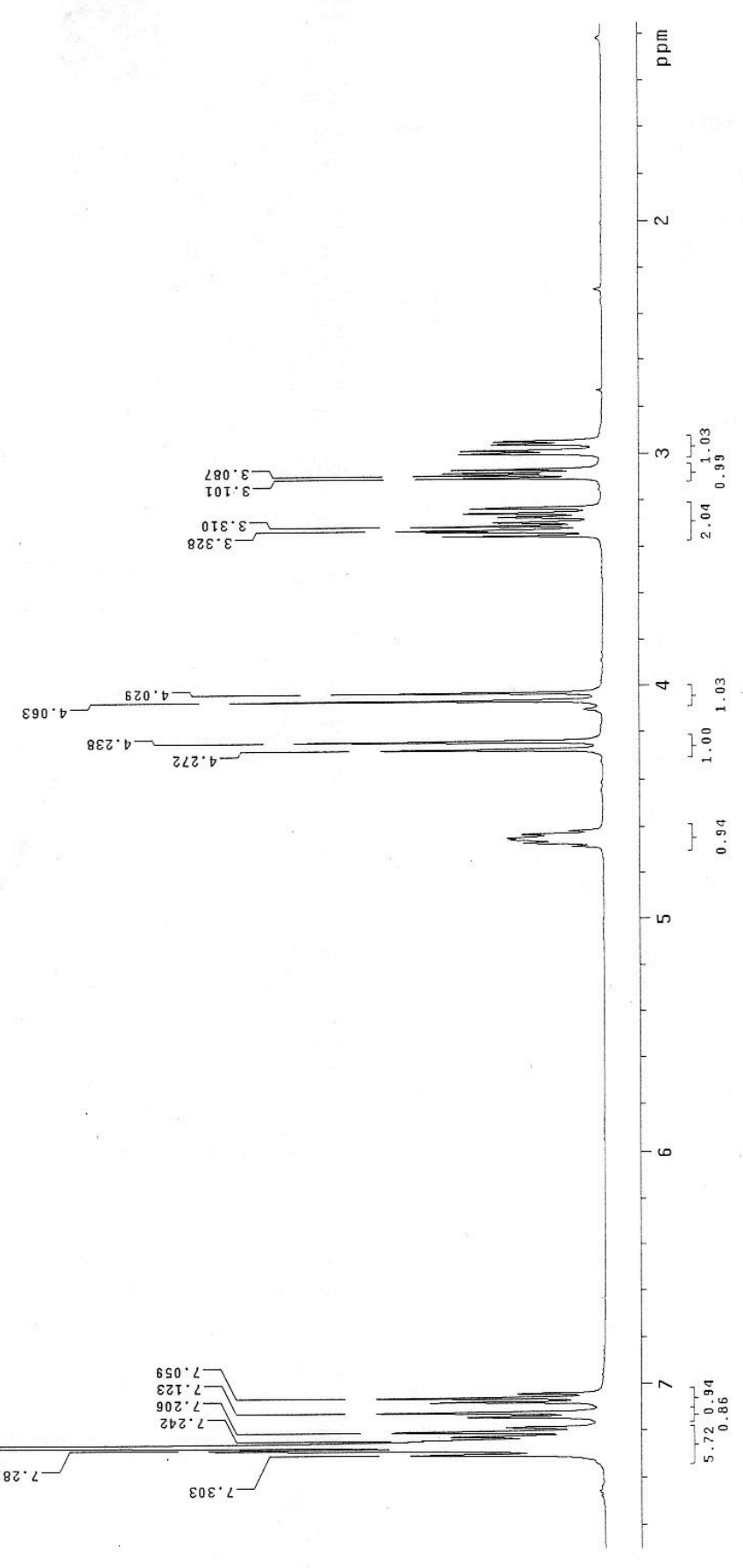


S 14

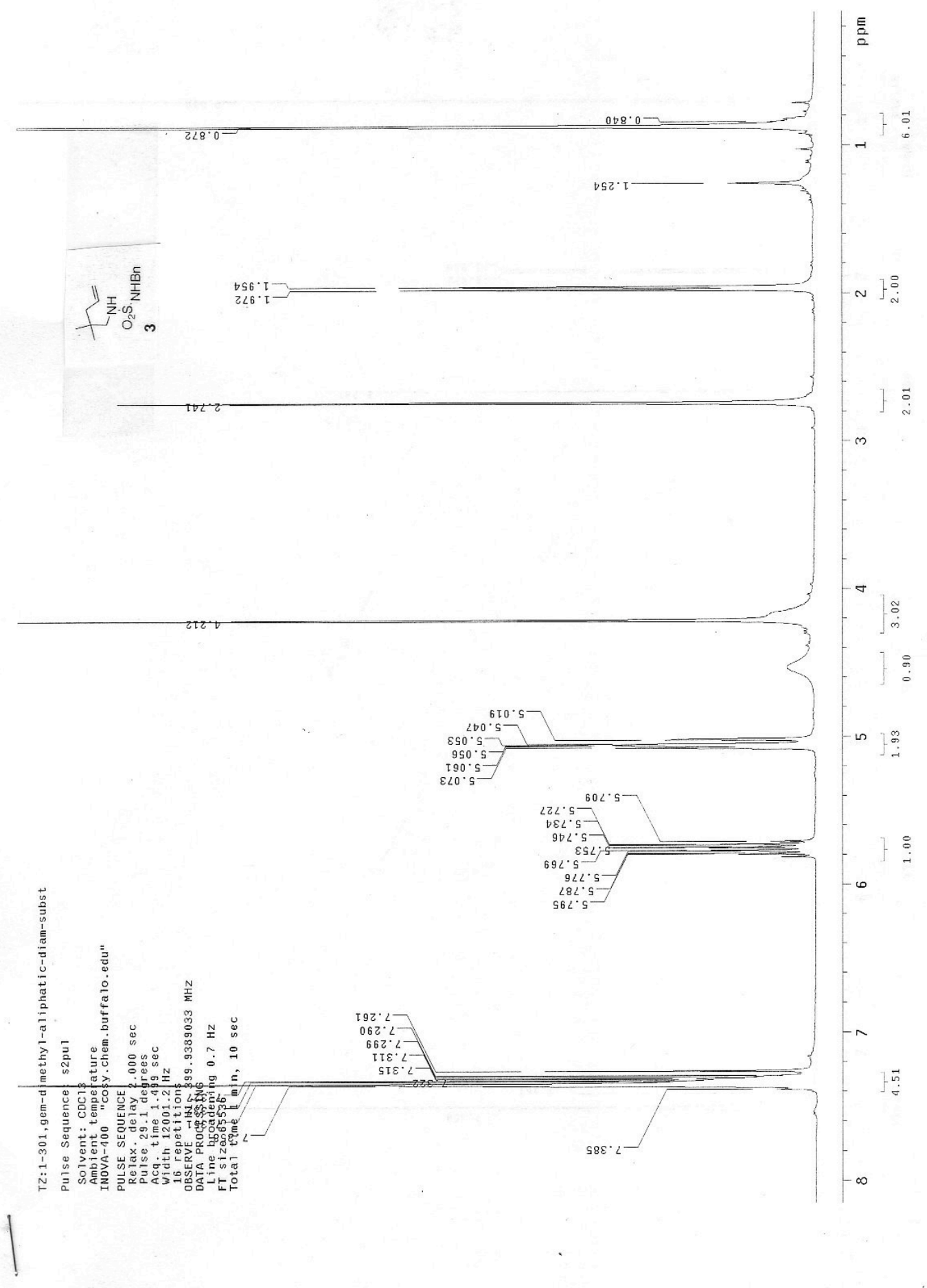


S 15
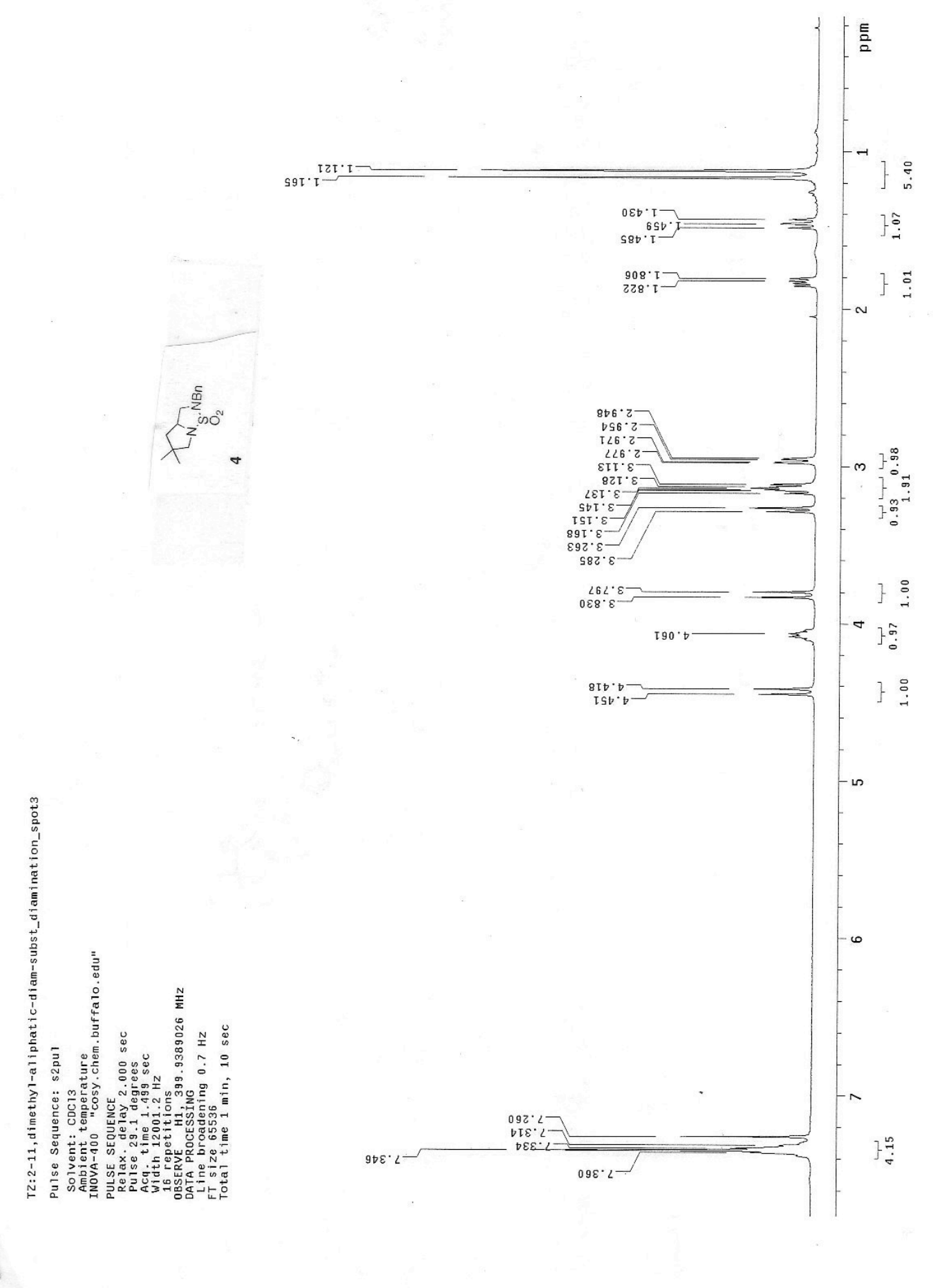
S 16

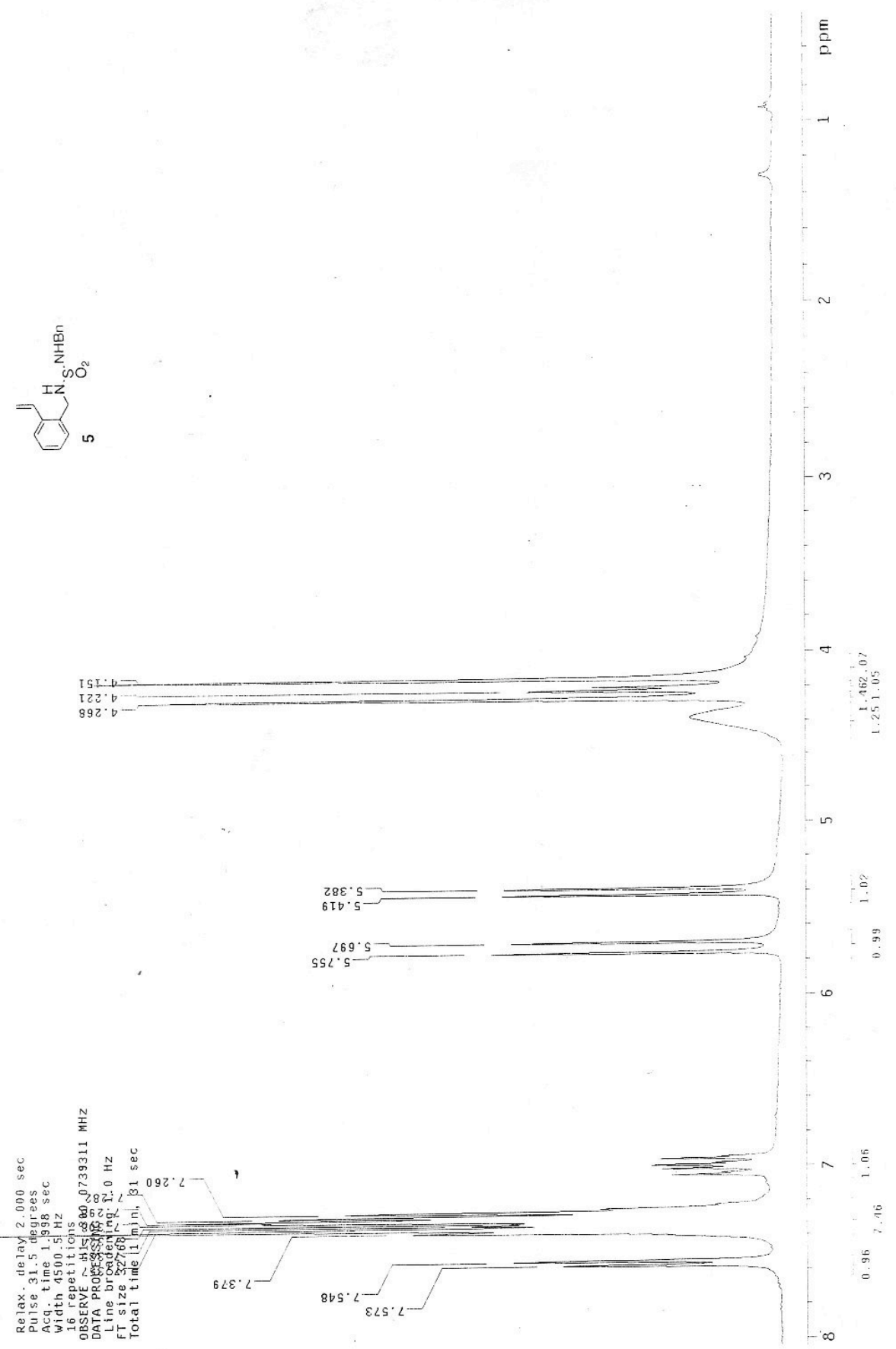



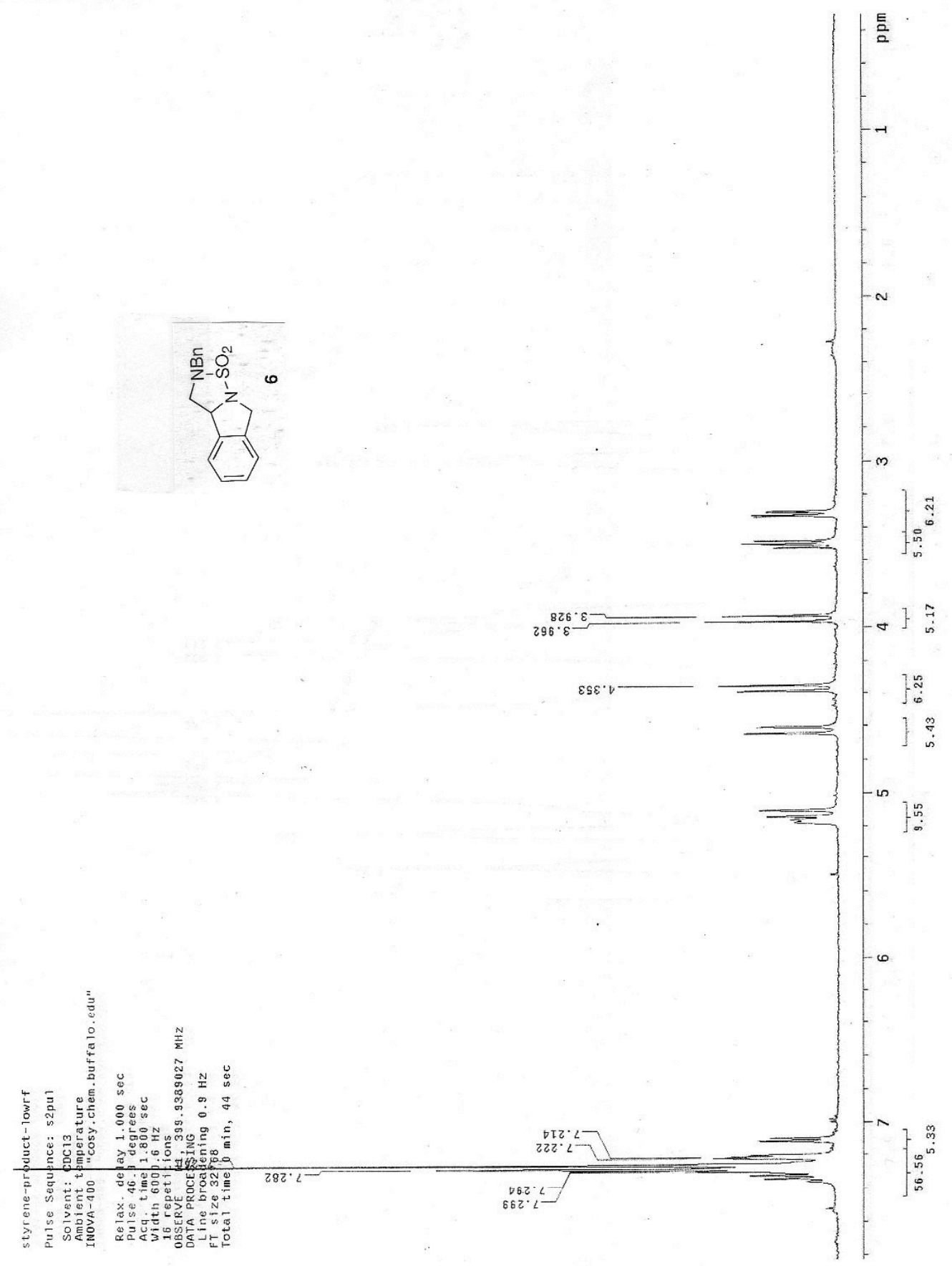

1 
S 18

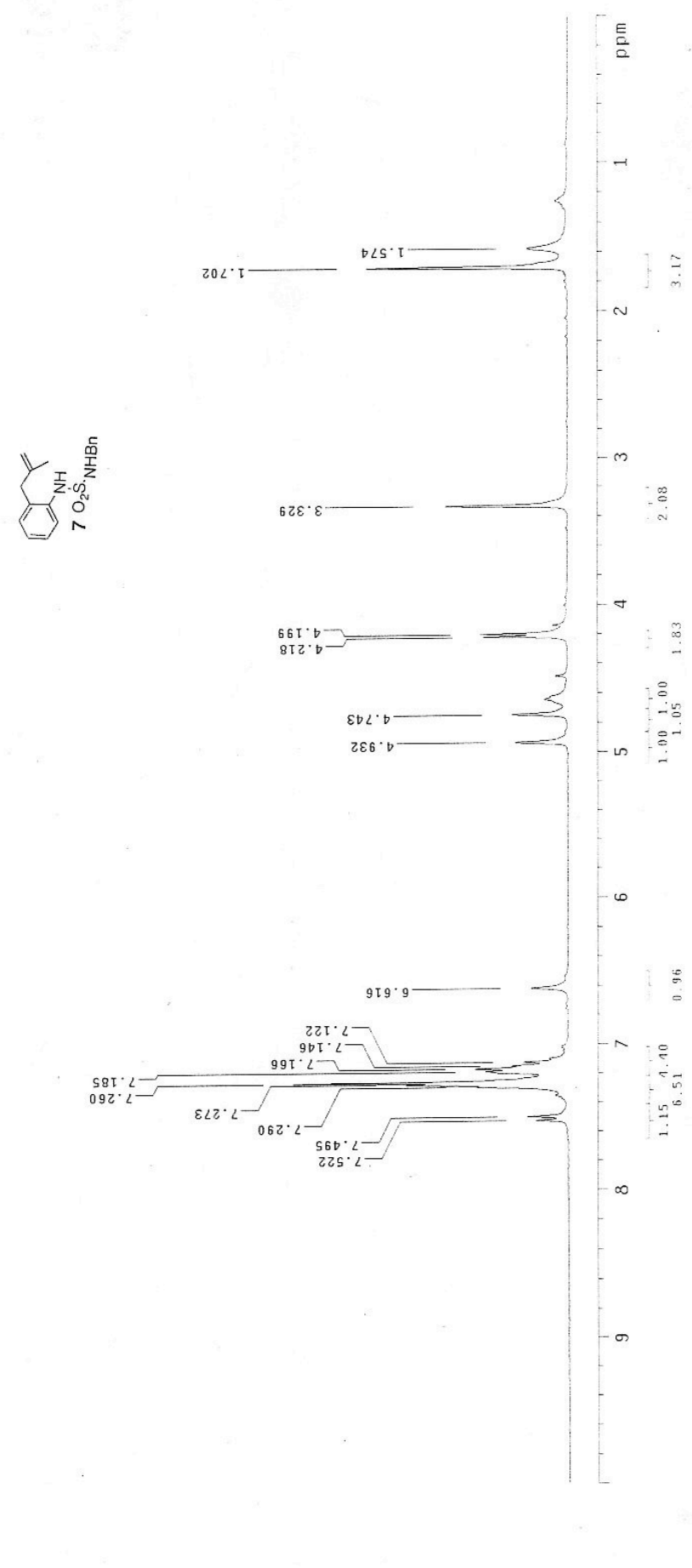




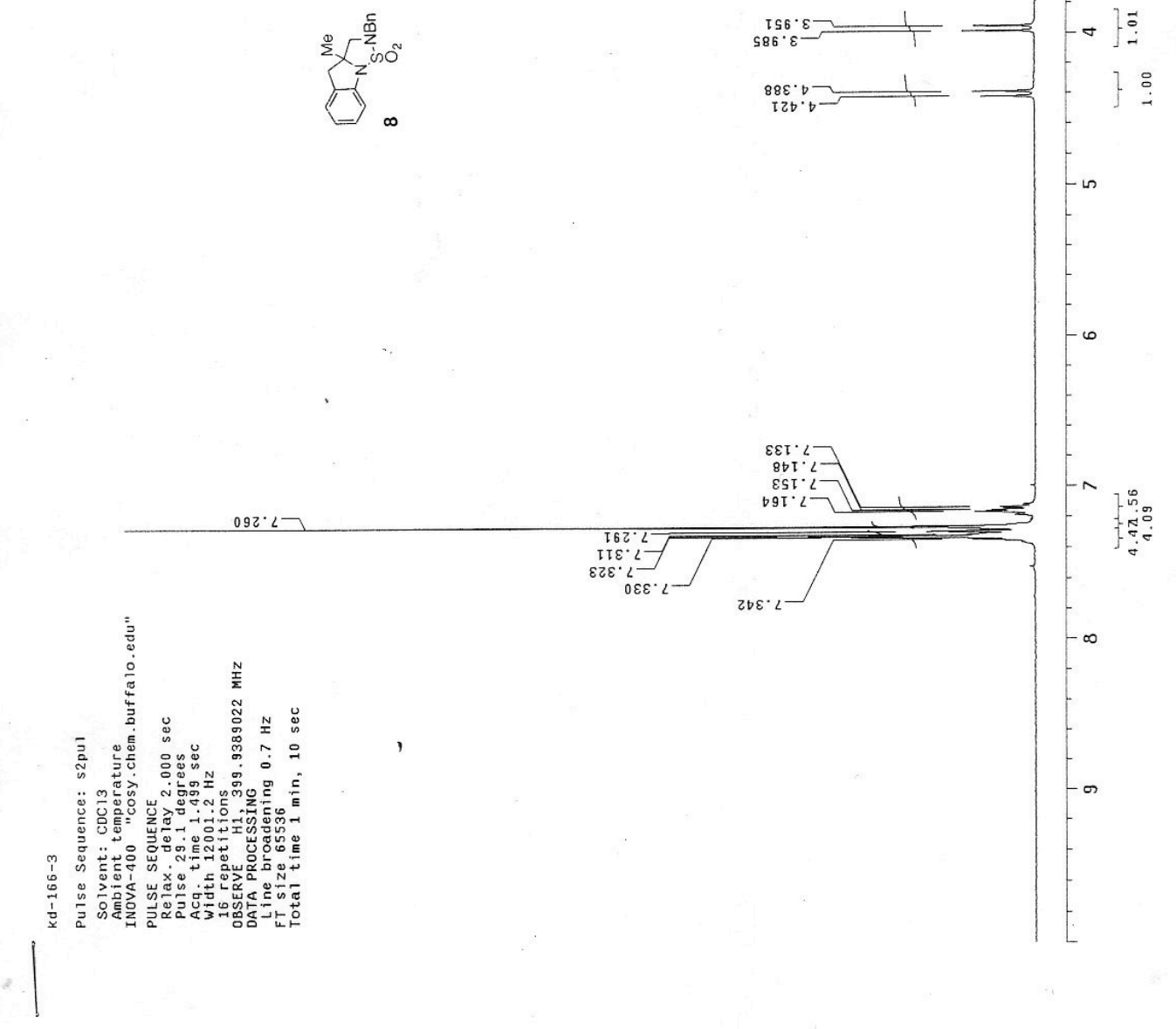




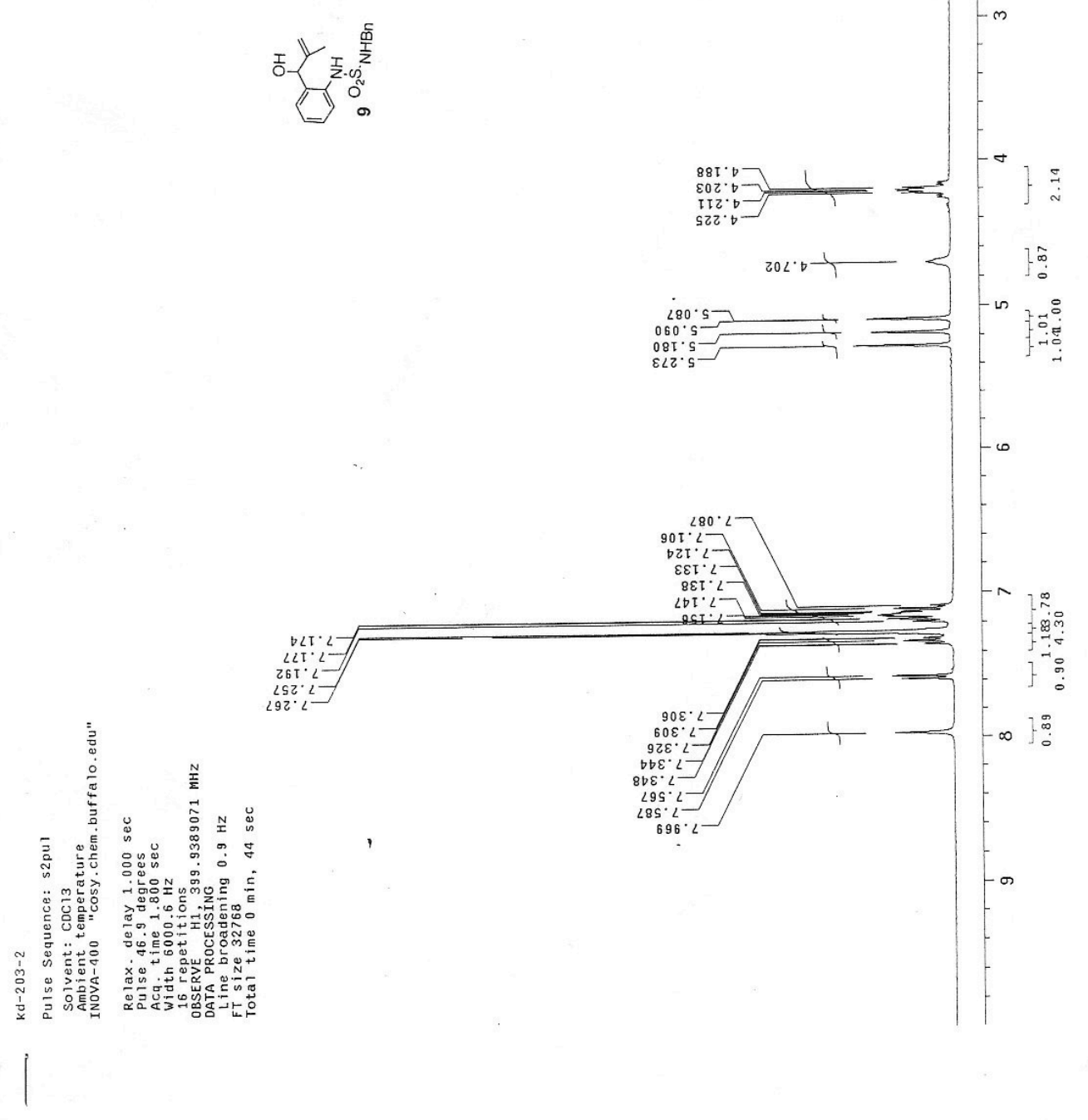


S 21

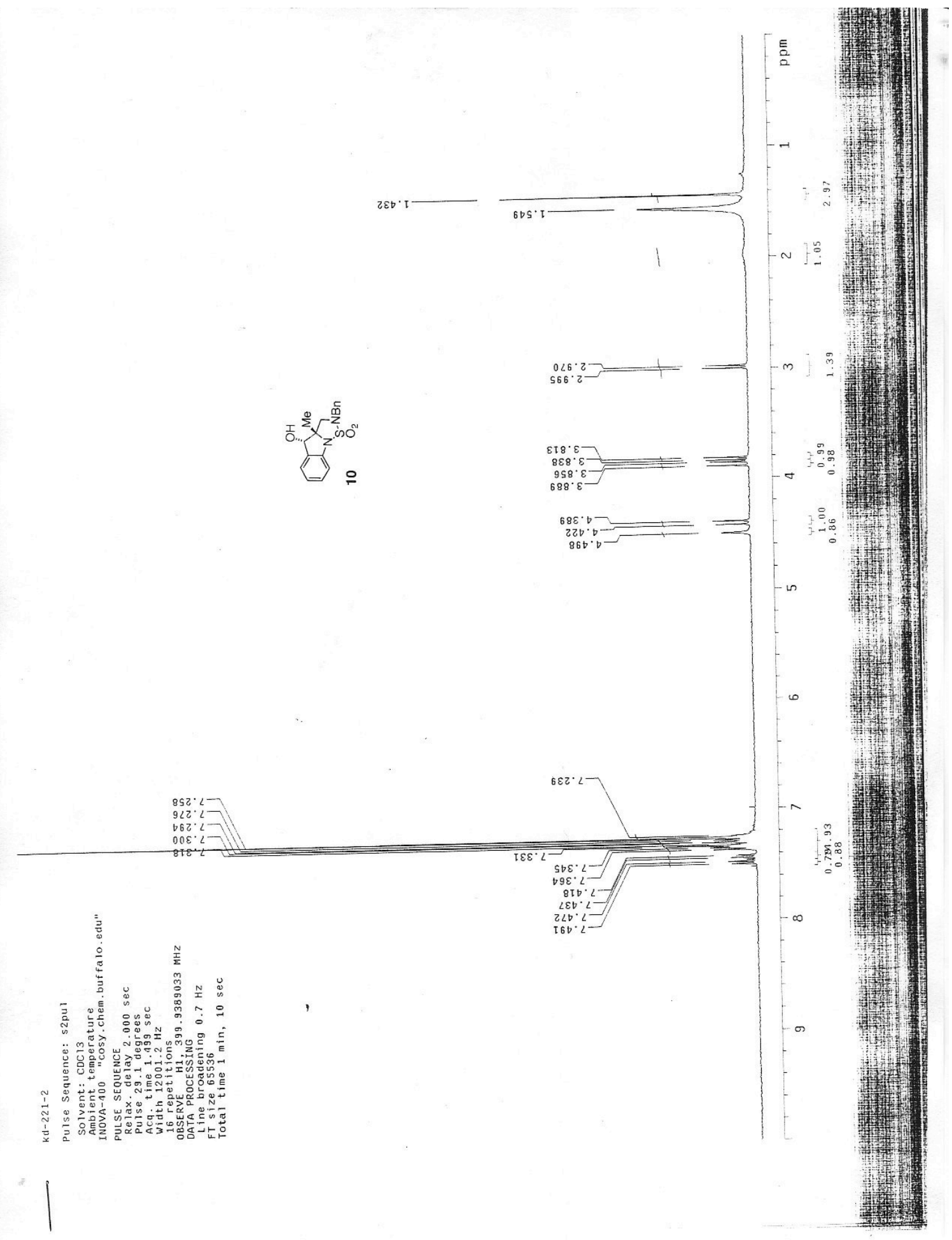


S 22

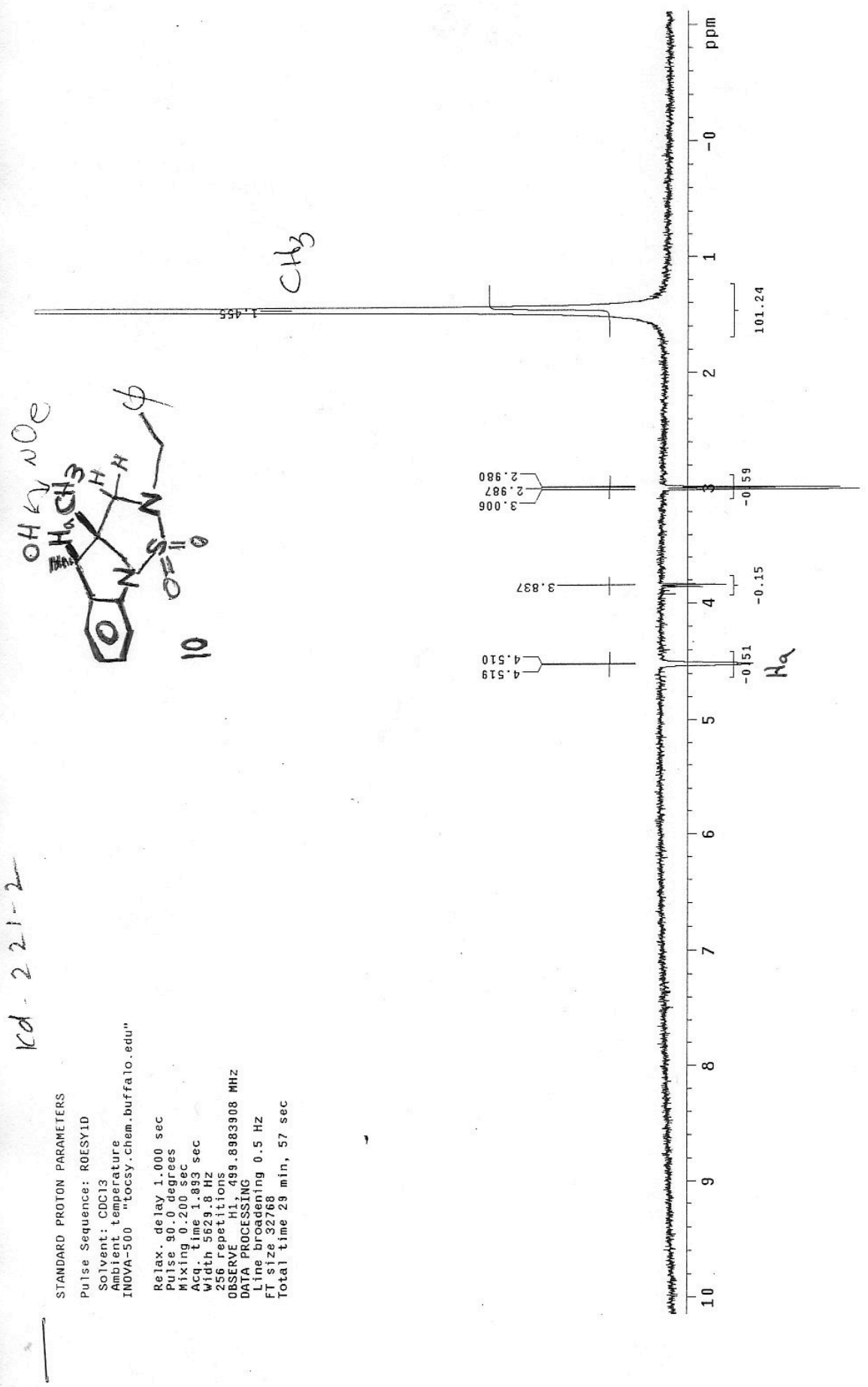




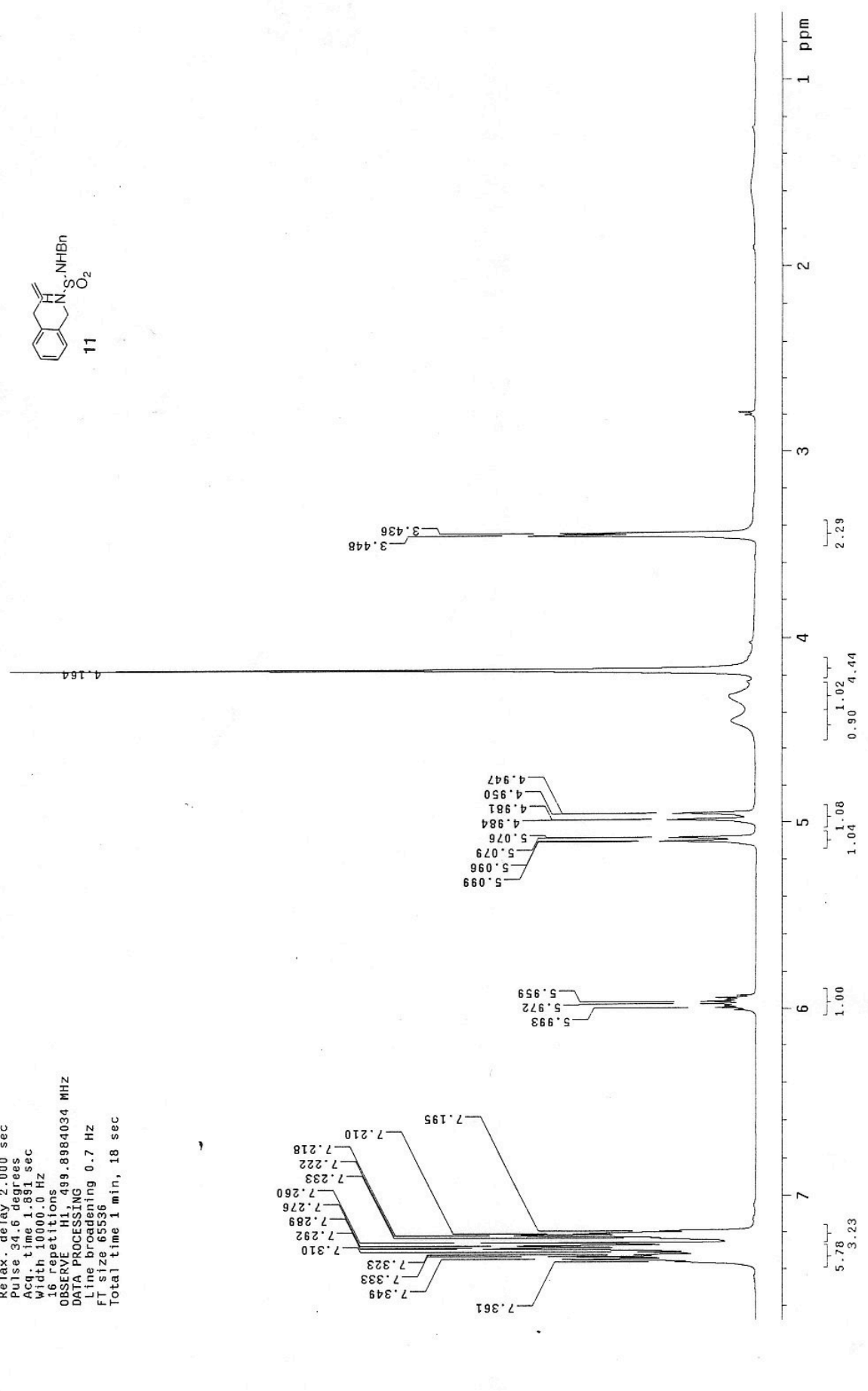



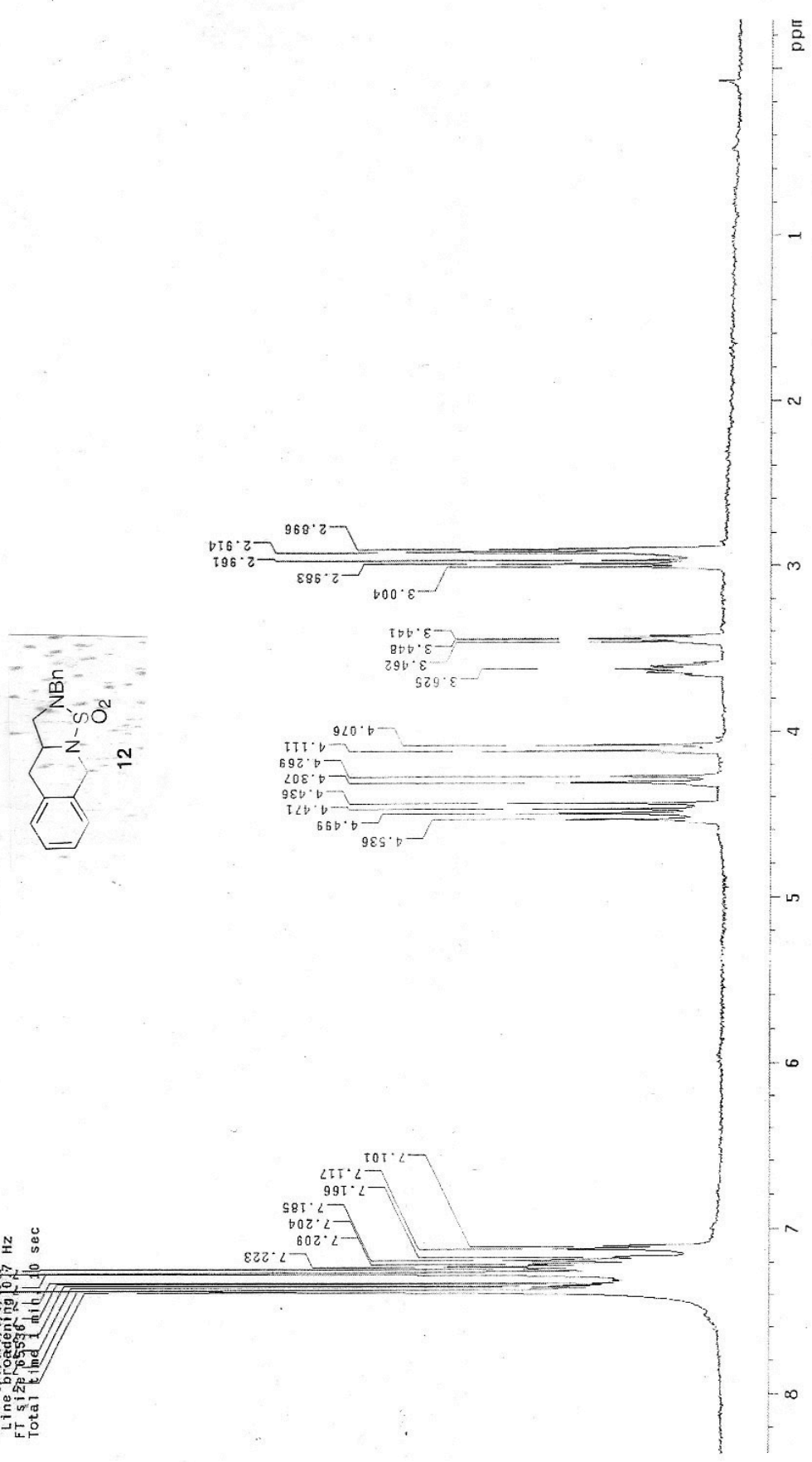

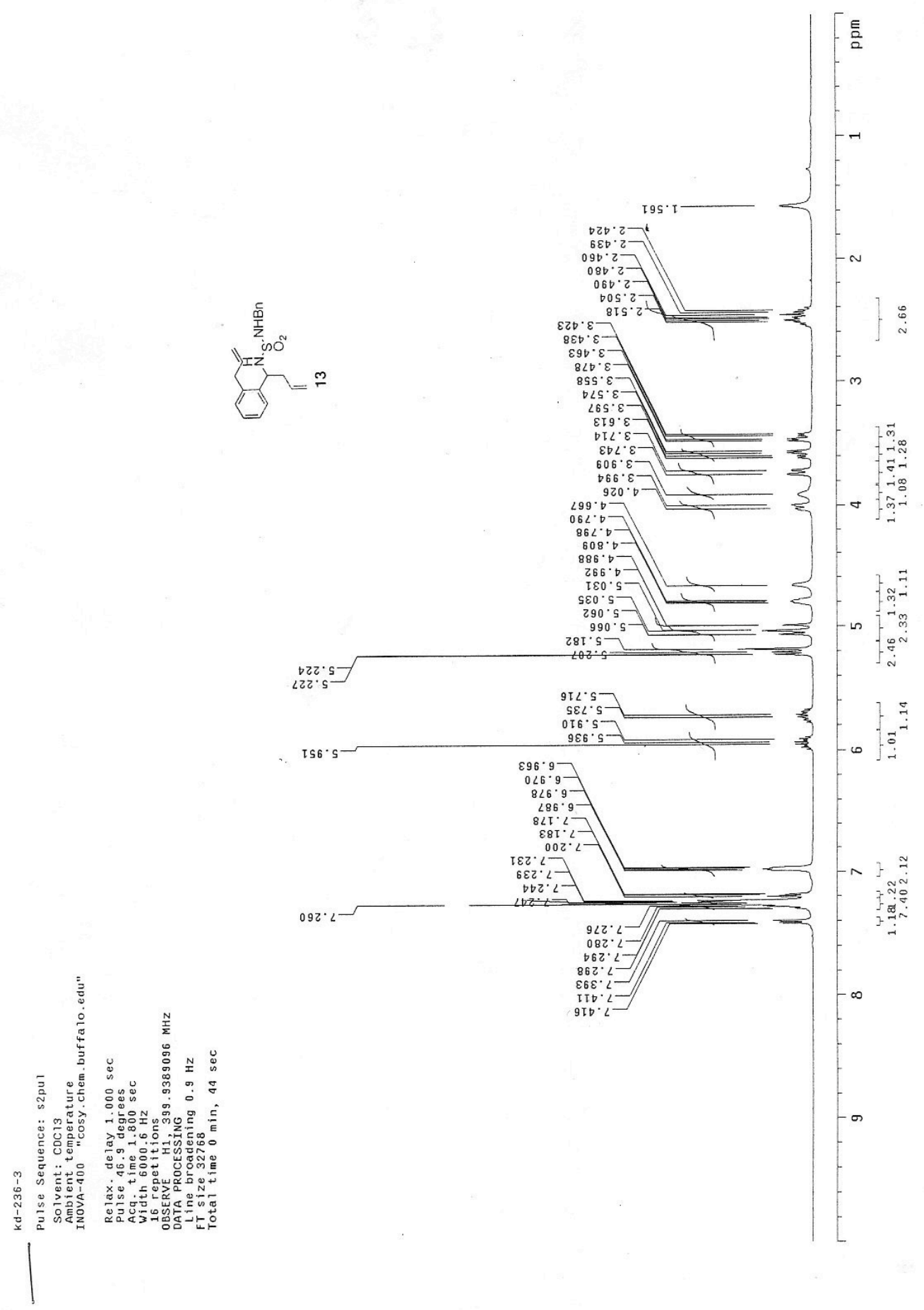

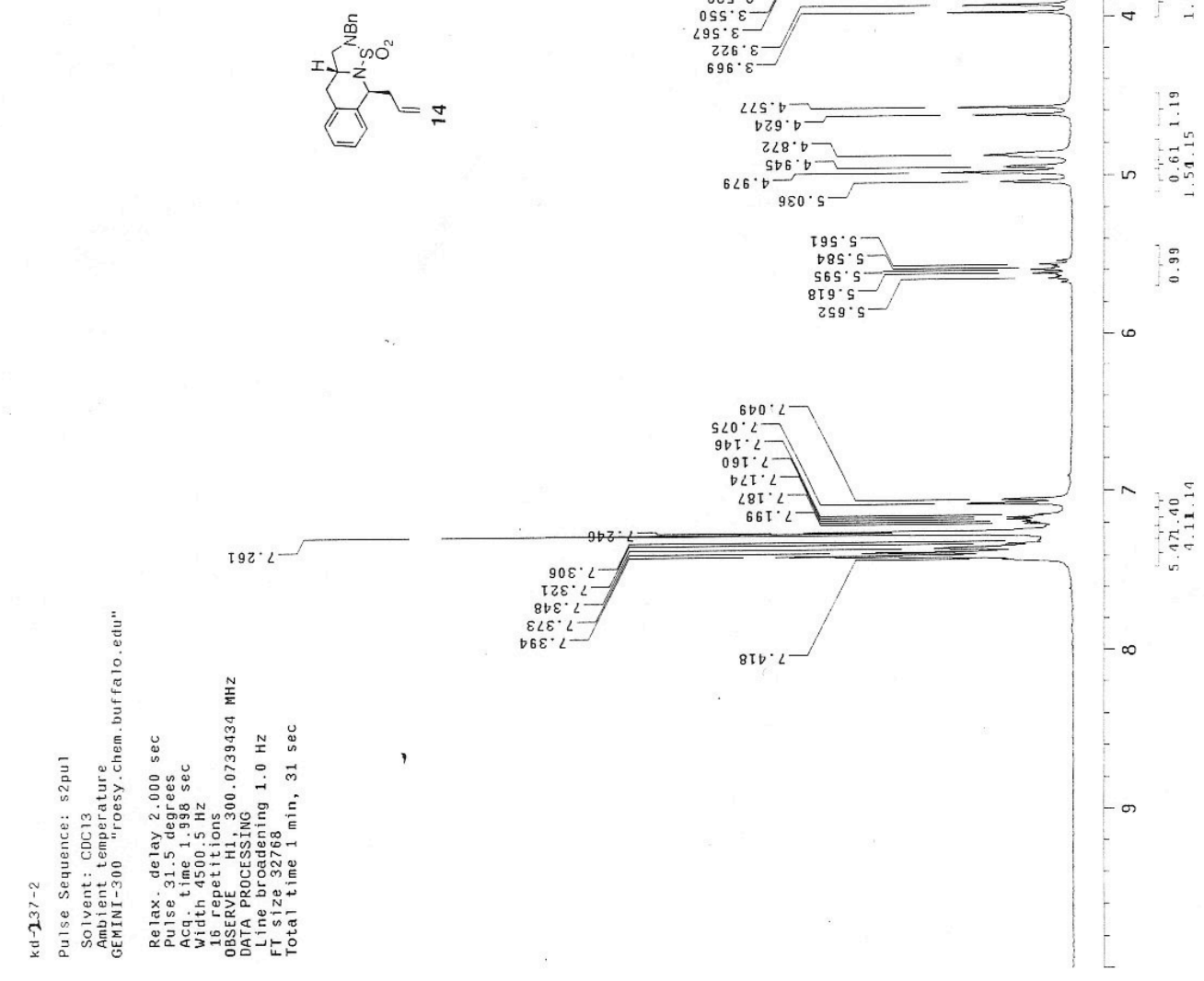

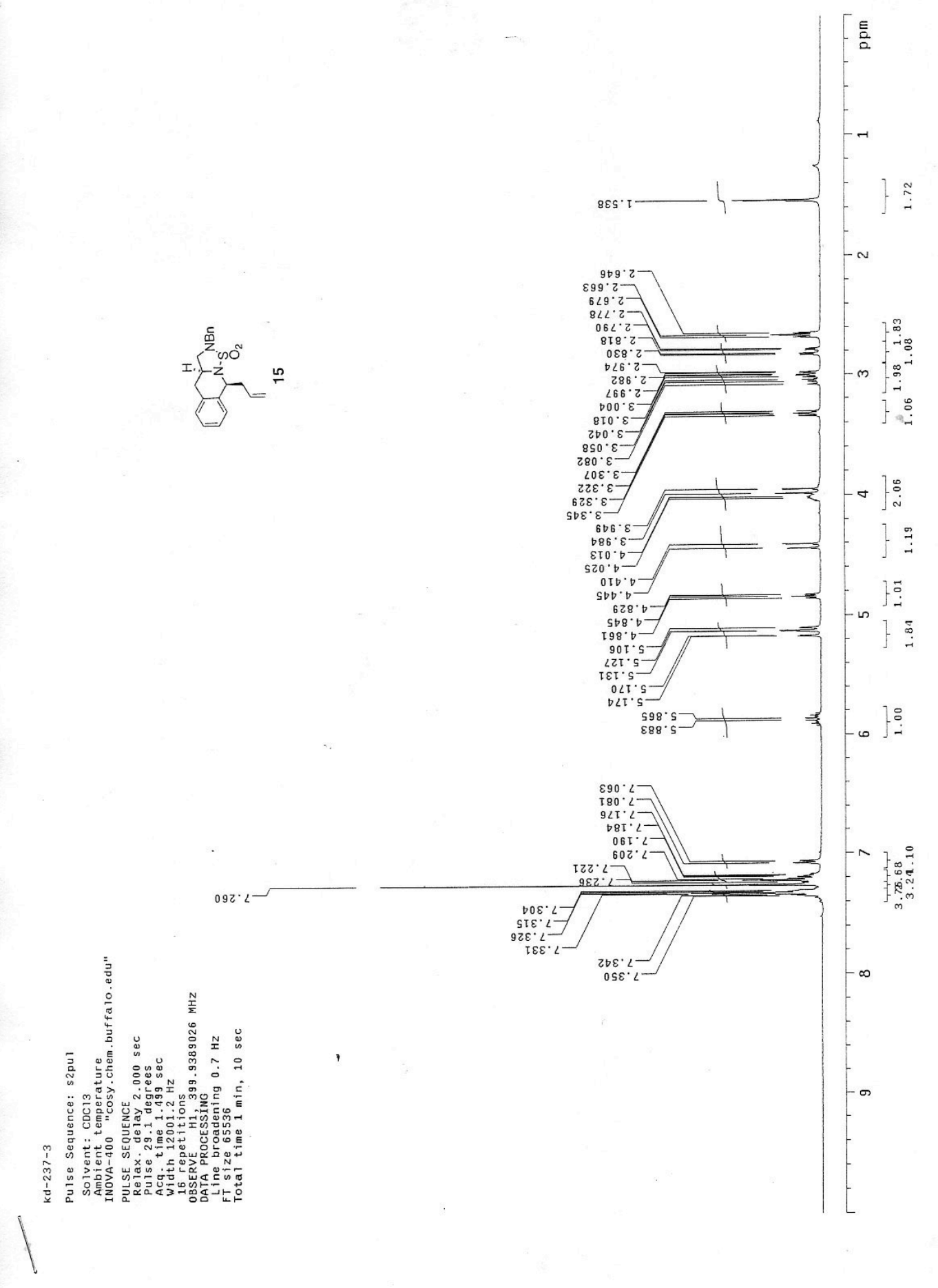


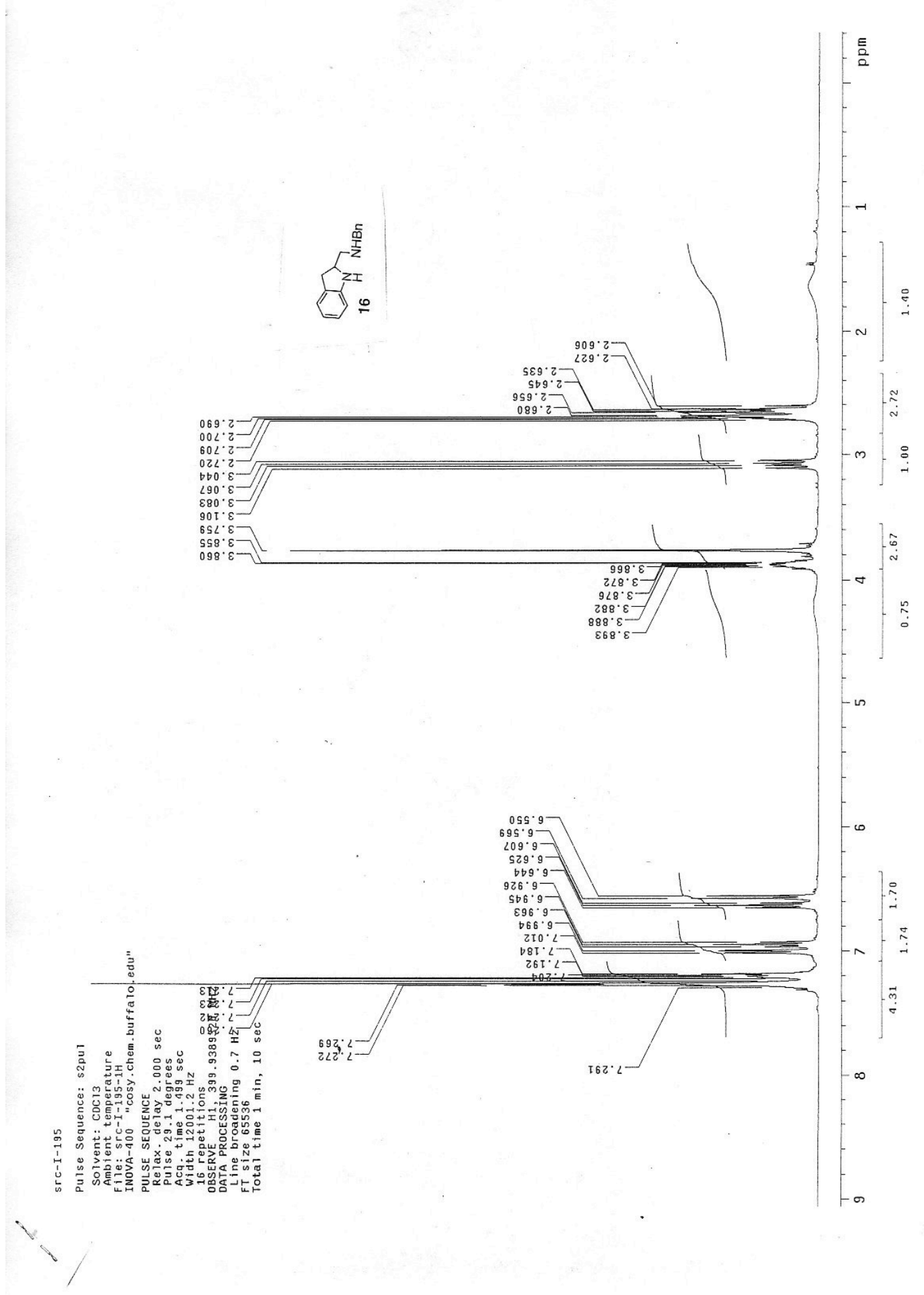

